\title{
Actuator Fault Detection for Discrete-Time Descriptor Systems via a Convex Unknown Input Observer with Unknown Scheduling Variables
}

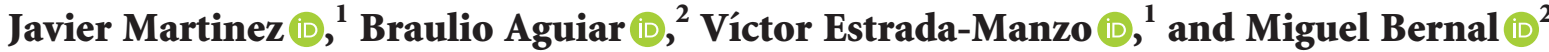 \\ ${ }^{1}$ Dept. of Mechatronics, Universidad Politécnica de Pachuca, C.P. 43830 Zempoala, Mexico \\ ${ }^{2}$ Dept. of Electrical and Electronics Engineering, Sonora Institute of Technology, 5 de Febrero 818 Sur, \\ C.P. 85000 Cd Obregon, Mexico
}

Correspondence should be addressed to Víctor Estrada-Manzo; victor_estrada@upp.edu.mx

Received 11 December 2020; Revised 13 February 2021; Accepted 20 February 2021; Published 30 March 2021

Academic Editor: Zhifeng Dai

Copyright ( $\odot 2021$ Javier Martinez et al. This is an open access article distributed under the Creative Commons Attribution License, which permits unrestricted use, distribution, and reproduction in any medium, provided the original work is properly cited.

This paper presents actuator fault detection of discrete-time nonlinear descriptor systems by means of nonlinear unknown input observers. The approach is based on the exact factorization of the estimation error in order to overcome the well-known problem of unmeasurable scheduling variables within the observation of convex models, thus avoiding the use of Lipschitz constants, differential mean value theorem, or robust techniques. As a result, the designing conditions are cast in terms of linear matrix inequalities and efficiently solved via commercially available software. Numerical as well as academic setups are provided to illustrate the advantages and performance of the proposal.

\section{Introduction}

The use of state observers [1] is important for many tasks within control theory. In particular, unknown input (UI) observers whose task is to estimate both the state and unknown inputs (they may be disturbances) have been developed in [2], and they are of particular interest also for fault detection and isolation $[3,4]$ or fault tolerant control schemes [5, 6]; they can be also applied in other fields [7]. For linear systems, there are several UI observers, e.g., [8-10]; for nonlinear systems, linear methods are mostly chosen because of its simplicity [11]. Nonlinear techniques such as sliding mode [12], adaptive schemes [13], Lipschitz approaches [14], high-gain observers [15], or combinations of them require certain structure of the model or performing nonlinear transformations.

On the other hand, convex models (a convex model is a collection of linear models interconnected by scalar functions (also known as scheduling functions), which are nonlinear and hold the convex sum property in a region $[16,17]$. If the convex model is the result of the sector nonlinearity [18], it is an exact representation of the nonlinear system) $[19,20]$ have been directly combined with the direct Lyapunov method; thus obtaining conditions in terms of linear matrix inequalities (LMIs) [21]. Conditions in the form of LMIs are preferred since they are numerically solvable via convex optimization techniques [22]. Within this context, there exist several state observers for both continuous [23, 24] and discrete-time systems [25-27]. In the case of UI observers, there are some works concerning proportional-integral setups $[28,29]$ or non-Luenberger forms [30-34].

Nevertheless, within the convex framework, the observer design presents an open problem: if the scheduling variables do not exclusively depend on measurable/available signals, the designing conditions get involve and difficult to cast as LMIs. Recent works have intended to tackle this issue by employing Lipschitz constraints [24,35], the differential mean value theorem (DMVT) $[36,37]$, and robust $H_{\infty}$ approaches to mitigate the influence of the unknown scheduling parameters [38]; more recently, in [39, 40], a transformation that enlarges the size of the state is proposed. 
In this work, we follow the ideas of [41] for solving this problem: algebraic rearrangements in order to properly factorize the error signal.

A generalized representation for standard state-space models is given by descriptor systems [42]; within this setup, a special case considers that the descriptor matrix is invertible [43]. For this class of descriptor systems, in [27], a convex observer design has been developed, and it considers only available scheduling variables; in $[44,45]$, an UI observer has been proposed, and it can handle unmeasurable scheduling variables by robust argumentations.

The estimation of the states and parameters makes possible the fault diagnosis, which is divided into fault detection, fault isolation, and fault identification [46, 47]. The fault detection (FD) is used to identify when there is a malfunction in the system and determine the moment when the fault occurs [48]. In FD, there exist some results using the UI observer for actuator fault, for example, [13] where an adaptive approach is developed for an aircraft actuator fault. In [49], UI observers are employed for fault detection and isolation.

Contributions: a novel convex UI observer scheme is used for discrete-time descriptor models with unmeasurable scheduling variables; the scheme makes use of algebraic manipulations instead of Lipschitz constraints, the differential mean value theorem, or robust techniques in order to obtain an adequate estimation error dynamics, thus relaxing the results. Actuator fault detection and estimation is performed by means of the proposed UI observer.

The rest of the paper is organized as follows: Section 2 provides the background for further developments and notation. Section 3 states the LMI conditions for the nonlinear UI observer design via convex models. In Section 4, the UI observer is applied to actuator fault detection and estimation of nonlinear systems. Section 5 illustrates the proposal via the numerical example and train system. Section 6 concludes the paper by giving some final remarks.

\section{Problem Statement}

Consider the following discrete-time nonlinear descriptor model:

$$
E\left(y_{k}\right) x_{k+1}=A\left(x_{k}\right) x_{k}+B\left(y_{k}\right) u_{k}+D\left(y_{k}\right) d_{k}, \quad y_{k}=C\left(x_{k}\right) x_{k},
$$

where $x_{k} \in \mathbb{R}^{n}$ is the state vector, $u_{k} \in \mathbb{R}^{m}$ is the input vector, and $d_{k} \in \mathbb{R}^{q}$ is the unknown input vector, $y_{k} \in \mathbb{R}^{o}$ is the output vector; $A\left(x_{k}\right), B\left(y_{k}\right), C\left(x_{k}\right), D\left(y_{k}\right)$, and $E\left(y_{k}\right)$ are matrix functions whose entries are smooth and bounded in a region $\Omega_{x} \subset \mathbb{R}^{n}$ including the origin. This work only considers the particular case when $E(\cdot)$ is full rank (the case when descriptor matrix is not invertible has been recently treated in $[50,51]$, this case is also referred as differential-algebraicequation (DAE) systems or singular ones [52], and it is out of the scope of this work. Fault diagnosis schemes for this type of systems have been addressed in [53]) for $x_{k} \in \Omega_{x}$; that is, from (1), it is always possible to obtain a standard state-space representation:

$$
\begin{aligned}
x_{k+1} & =E^{-1}\left(y_{k}\right)\left(A\left(x_{k}\right) x_{k}+B\left(y_{k}\right) u_{k}+D\left(y_{k}\right) d_{k}\right) \\
& =f\left(x_{k}, u_{k}, d_{k}\right) .
\end{aligned}
$$

In what follows, arguments will be omitted when their meaning can be inferred from the context.

The approach is based on designing an observer for the estimation of both the state $x_{k}$ and the unknown input $d_{k}$; to this end, an augmented vector is employed $[29,44]$, that is, $\chi_{k}=\left[\begin{array}{ll}x_{k}^{T} & \mathbf{d}_{k}^{T}\end{array}\right]^{T}, \quad \mathbf{d}_{k}=\left[\begin{array}{llll}d_{k}^{T} & d_{k+1}^{T} & \ldots & d_{k+p}^{T}\end{array}\right]^{T} \in \mathbb{R}^{(p+1) q}$, where $p$ is such that $(z-1)^{p+1} d_{k} \approx 0$ as proposed in [45]; for instance, for $p=1$, we have

$$
\mathbf{d}_{\mathbf{k}+\mathbf{1}}=S \mathbf{d}_{k} \text {, with } \mathbf{d}_{\mathbf{k}+\mathbf{1}}=\left[\begin{array}{l}
d_{k+1} \\
d_{k+2}
\end{array}\right], S=\left[\begin{array}{cc}
0 & 1 \\
-1 & 2
\end{array}\right] .
$$

Hence, an augmented system yields

$$
\mathscr{E}\left(y_{k}\right) \chi_{k+1}=\mathscr{A}\left(x_{k}, y_{k}\right) \chi_{k}+\mathscr{B}\left(y_{k}\right) u_{k}, \quad y_{k}=\mathscr{C}\left(x_{k}\right) \chi_{k},
$$

where $\chi \in \mathbb{R}^{n+(p+1) q}, S \in \mathbb{R}^{(p q+q) \times(p q+q)}$ is a known matrix, and

$$
\begin{aligned}
\mathscr{E}\left(y_{k}\right) & =\left[\begin{array}{cc}
E\left(y_{k}\right) & 0 \\
0 & I
\end{array}\right], \\
\mathscr{A}\left(x_{k}, y_{k}\right) & =\left[\begin{array}{c|c}
A\left(x_{k}\right) & D\left(y_{k}\right) 0_{n \times p q} \\
\hline 0 & S
\end{array}\right], \\
\mathscr{B}\left(y_{k}\right) & =\left[\begin{array}{c}
B\left(y_{k}\right) \\
0
\end{array}\right], \\
\mathscr{C}\left(x_{k}\right) & =\left[\begin{array}{ll}
C\left(x_{k}\right) & 0
\end{array}\right] .
\end{aligned}
$$

In the literature, most of the observer design approaches deal with systems in standard form (2); for instance, without the unknown input, $x_{k+1}=f\left(x_{k}, u_{k}\right)$ and $y_{k}=C x_{k}$; they consider special cases $x_{k+1}=A x_{k}+$ $B f\left(y_{k}, u_{k}\right)+\phi\left(x_{k}\right)$; thus, the task is to stabilize an error system with the form $e_{k+1}=(A-L C) e_{k}+\phi\left(x_{k}\right)-\phi\left(\hat{x}_{k}\right)$, where $e_{k}=x_{k}-\hat{x}_{k}$ is the estimation error, $\hat{x}_{k}$ is the estimated state vector, $L$ is the observer gain to be designed, and the function $\phi(\cdot)$ is assumed to hold Lipschitz bounds, i.e., $\|\phi(x)-\phi(\widehat{x})\| \leq\|\mathscr{L} x-\hat{x}\|, \mathscr{L}>0$ being a Lipschitz constant $[54,55]$. In the context of convex models, most of the works only consider that the scheduling variables are available and then $\phi\left(x_{k}\right)-\phi\left(\hat{x}_{k}\right)=0$ [25], which, in practice, is not realistic. For the general case, when $\phi\left(x_{k}\right)-\phi\left(\hat{x}_{k}\right) \neq 0$, this expression is treated by means for Lipschitz bounds [24], as a perturbation via $H_{\infty}$ approach [38], as an uncertainty via robust approaches [56]; other works employ the differential mean value theorem [37], Jacobian [57], or transformations that enlarge the size of the state [40]. Nevertheless, these approaches are conservative approximations, or increase the computational complexity of the problem, or are only valid for particular cases [58]; additionally, none of them consider observers for systems of form (1). Next section presents a methodology that avoids the use of previous ones. 
2.1. An Amenable Descriptor Error Form. In order to obtain an amenable error system for Lyapunov analysis, in [41], a methodology that avoids the use of Lipschitz-like bounds has been presented; it is based on algebraic operations in order to factorize the estimation error at the left side of error dynamic difference equation. Thus, the variation of the
Lyapunov function along the trajectories of the error system can be written as $\Delta V(e)=e_{k}^{T} Q(\cdot) e_{k}$ and $\Delta V(e)<0$ if $Q(\cdot)<0$, and the latter is guaranteed via convex models and linear matrix inequalities. Motivated by these ideas, the following observer structure is adopted:

$$
\mathscr{E}\left(y_{k}\right) \hat{\chi}_{k+1}=\mathscr{A}\left(\hat{x}_{k}, y_{k}\right) \widehat{\chi}_{k}+\mathscr{B}\left(y_{k}\right) u_{k}+L\left(\hat{x}_{k}, y_{k}\right)\left(y_{k}-\hat{y}_{k}\right), \quad \hat{y}_{k}=\mathscr{C}\left(\hat{x}_{k}\right) \widehat{\chi}_{k} .
$$

with

$$
\begin{aligned}
\hat{x}_{k} & =\left[\begin{array}{l}
\hat{x}_{k} \\
\widehat{\mathbf{d}}_{\mathbf{k}}
\end{array}\right], \\
\mathscr{A}\left(\hat{x}_{k}, y_{k}\right) & =\left[\begin{array}{c|c}
A\left(\hat{x}_{k}\right) & D\left(y_{k}\right) 0_{n \times p q} \\
\hline 0 & S
\end{array}\right], \\
\mathscr{C}\left(\hat{x}_{k}\right) & =\left[\begin{array}{ll}
C\left(\hat{x}_{k}\right) & 0
\end{array}\right],
\end{aligned}
$$

and the nonlinear observer gain $L\left(\widehat{x}_{k}, y_{k}\right) \in \mathbb{R}^{(n+p q+q) \times o}$ depending only on all the available signals; it should be designed such that the estimation error

$$
e_{k}=\chi_{k}-\widehat{\chi}_{k}=\left[\begin{array}{c}
x_{k}-\widehat{x}_{k} \\
\mathbf{d}_{\mathbf{k}}-\widehat{d}_{\mathbf{k}}
\end{array}\right]
$$

satisfies $\lim e_{k}=0$. Thus, in [41], it has been proven that, under mild assumptions, it is always possible to write the error dynamics as

$$
\mathscr{E}\left(y_{k}\right) e_{k+1}=\left(\overline{\mathscr{A}}\left(x_{k}, \hat{x}_{k}\right)-L\left(\hat{x}_{k}, y_{k}\right) \overline{\mathscr{C}}\left(x_{k}, \hat{x}_{k}\right)\right) e_{k},
$$

where $\overline{\mathscr{A}}\left(x_{k}, \hat{x}_{k}\right) e_{k}=\mathscr{A}\left(x_{k}, y_{k}\right) \chi_{k}-\mathscr{A}\left(\hat{x}_{k}, y_{k}\right) \hat{\chi}_{k} \quad$ and $\overline{\mathscr{C}}\left(x_{k}, \hat{x}_{k}\right) e_{k}=\mathscr{C}(x) \chi_{k}-\mathscr{C}(\hat{x}) \widehat{\chi}_{k}$ have bounded entries in $\Omega_{x} \times \Omega_{\widehat{x}}$. For instance, consider a polynomial expression $p(x)-p(\widehat{x})$ with $p(x)=x_{1} x_{2}$ and $p(\widehat{x})=\widehat{x}_{1} \widehat{x}_{2}$; following [41], we have $p(x)-p(\hat{x})=0.5\left(x_{2}+\widehat{x}_{2}\right) e_{1}+0.5$ $\left(x_{1}+\hat{x}_{1}\right) e_{2}, \quad e_{1}=x_{1}-\hat{x}_{1}, e_{2}=x_{2}-\hat{x}_{2}$. Now, consider a nonpolynomial expression $\widetilde{p}(x)-\widetilde{p}(\widehat{x})$, with $\widetilde{p}(x)=\sin x_{1}$ and $\tilde{p}(\widehat{x})=\sin \widehat{x}_{1}$; a third-order Taylor approximation will give $\widetilde{p}(x)-\tilde{p}(\hat{x}) \approx x_{1}-\left(x_{1}^{3} / 6\right)-\widehat{x}_{1}+\left(\hat{x}_{1}^{3} / 6\right)$, and then we can apply similar procedure as before.

Now, error system (9) has an amenable form for Lyapunov-based analysis. However, if the aim is to obtain LMI conditions, (9) should be expressed as an exact convex model, and this is the matter of the following section.

2.2. Convex Expressions. The sector nonlinearity [18] is employed to express bounded nonconstant terms $z(\cdot) \in\left[z^{0}, z^{1}\right]$ as a convex sums of its bounds, that is, $z(\cdot)=w_{0}(z) z^{0}+w_{1}(z) z^{1}$, where $z^{0}$ and $z^{1}$ are the minimum and maximum of $z(\cdot)$ in a region; the functions $w_{0}=$ $\left(z^{1}-z(\cdot)\right) /\left(z^{1}-z^{0}\right)$ and $w_{1}=1-w_{0}$ hold the convex sum property in the same modeling region, i.e., $w_{0}(z)+w_{1}(z)=$ 1 and $w_{0}, w_{1} \in[0,1]$.

Note that, in (9), matrices $\mathscr{E}\left(y_{k}\right), \overline{\mathscr{A}}\left(x_{k}, \widehat{x}_{k}\right)$, and $\overline{\mathscr{C}}\left(x_{k}, \hat{x}_{k}\right)$ contain nonconstant terms depending on $x_{k}, \hat{x}_{k}$, and $y_{k}$; clearly, all the state variables are not fully available; thus, a useful convex rewriting must take this into account. The following steps extend the sector nonlinearity to our case:

Step 1: identify all the nonconstant terms, also known as scheduling variables, in $\mathscr{E}\left(y_{k}\right), \overline{\mathscr{A}}\left(x_{k}, \widehat{x}_{k}\right)$, and $\overline{\mathscr{C}}\left(x_{k}, \widehat{x}_{k}\right)$ depending exclusively on available signals, and capture them in the vector $z\left(\hat{x}_{k}, y_{k}\right) \in \mathbb{R}^{s}$ while all the rest of the terms should be grouped in $\zeta\left(x_{k}, \hat{x}_{k}, y_{k}\right) \in \mathbb{R}^{\sigma}$. Each entry is assumed to be bounded in $\Omega_{x} \times \Omega_{\widehat{x}}$, i.e., $z_{i}\left(\hat{x}_{k}, y_{k}\right) \in\left[z_{i}^{0}, z_{i}^{1}\right], \quad i \in\{1,2, \quad \ldots, s\}, \quad$ and $\zeta_{j}\left(x_{k}, \widehat{x}_{k}, y_{k}\right) \in\left[\zeta_{j}^{0}, \zeta_{j}^{1}\right], j \in\{1,2, \ldots, \sigma\}$.

Step 2: construct, for each $z_{i}\left(\widehat{x}_{k}, y_{k}\right), i \in\{1,2, \ldots, s\}$, and $\zeta_{j}\left(x_{k}, \hat{x}_{k}, y_{k}\right), j \in\{1,2, \ldots, \sigma\}$ a pair of scalar convex functions as follows:

$$
\begin{gathered}
w_{0}^{i}(\hat{x}, y)=\frac{z_{i}^{1}-z_{i}(\hat{x}, y)}{z_{i}^{1}-z_{i}^{0}}, \\
w_{1}^{i}(\hat{x}, y)=1-w_{0}^{i}(\widehat{x}, y), \\
\omega_{0}^{j}(x, \hat{x}, y)=\frac{\zeta_{j}^{1}-\zeta_{j}(x, \hat{x}, y)}{\zeta_{j}^{1}-\zeta_{j}^{0}}, \\
\omega_{1}^{j}(x, \hat{x}, y)=1-\omega_{0}^{j}(x, \hat{x}, y) ;
\end{gathered}
$$

by construction, these functions hold the convex sum property in $\Omega_{x} \times \Omega_{\widehat{x}}$, i.e., $w_{0}^{i}(\hat{x}, y)+w_{1}^{i}(\widehat{x}, y)=1$, $w_{0}^{i}(\hat{x}, y), w_{1}^{i}(\hat{x}, y) \in[0,1], \omega_{0}^{j}(x, \hat{x}, y)+\omega_{1}^{j}(x, \widehat{x}, y)=$ $1, \omega_{0}^{j}(x, \hat{x}, y), \omega_{1}^{j}(x, \hat{x}, y) \in[0,1]$.

Step 3: define the scheduling functions as

$$
\begin{aligned}
& \mathbf{w}_{i}(z)=w_{i_{1}}^{j}\left(z_{1}\right) w_{i_{2}}^{2}\left(z_{2}\right), \ldots, w_{i_{s}}^{s}\left(z_{s}\right), \\
& \boldsymbol{\omega}_{j}(\zeta)=\omega_{j_{1}}^{1}\left(\zeta_{1}\right) \omega_{j_{2}}^{2}\left(\zeta_{2}\right), \ldots, \omega_{j_{\sigma}}^{\sigma}\left(\zeta_{\sigma}\right),
\end{aligned}
$$

with $i \in\{1,2, \ldots, r\}, j \in\{1,2, \ldots, \rho\} i_{j} \in\{0,1\}, r=2^{s}$, $\rho=2^{\sigma}$; moreover, the sets of indexes $\left[i_{1} i_{2}, \ldots, i_{s}\right]$ and $\left[j_{1} j_{2}, \ldots, j_{\sigma}\right]$ are a $s$-digit and $\sigma$-digit binary representation of $(i-1)$ and $(j-1)$, respectively. The scheduling functions also hold the convex sum property in $\Omega_{x} \times \Omega_{\widehat{x}}$, that is, $\sum_{i=1}^{r} \mathbf{w}_{i}(z)=1, \mathbf{w}_{i}(z) \in[0,1]$, $\sum_{j=1}^{\rho} \omega_{j}(\zeta)=1$, and $\omega_{j}(\zeta) \in[0,1]$.

Step 4: compute the vertex models $\mathscr{E}_{i}=\left.\mathscr{E}\left(y_{k}\right)\right|_{\mathbf{w}_{i}=1}$, $\overline{\mathscr{A}}_{i j}=\left.\overline{\mathscr{A}}\left(x_{k}, \bar{x}_{k}\right)\right|_{\mathrm{w}_{i} \omega_{j}=1}, \overline{\mathscr{C}}_{i j}=\left.\overline{\mathscr{C}}\left(x_{k}, \widehat{x}_{k}\right)\right|_{\mathrm{w}_{i} \omega_{j}=1}, i \in\{1,2$, $\ldots, r\}, j \in\{1,2, \ldots, \rho\}$. 
Thus, an exact convex representation of (9) is $\sum_{i=1}^{r} \mathbf{w}_{i}\left(z_{k}\right) \mathscr{E}_{i} e_{k+1}=\sum_{i=1}^{r} \sum_{j=1}^{\rho} \mathbf{w}_{i}\left(z_{k}\right) \mathbf{w}_{j}\left(\zeta_{k}\right)\left(\overline{\mathscr{A}}_{i j}-L\left(\hat{x}_{k}, y_{k}\right) \overline{\mathscr{C}}_{i j}\right) e_{k}$.

The nonlinear observer gain is $L\left(\hat{x}_{k}, y_{k}\right)$ and will be defined later on.

2.2.1. Notation. For convex expressions, the following shorthand notation will be employed throughout the manuscript: single convex sums $\Upsilon_{\mathbf{w}\left(z_{k}\right)}=\sum_{i=1}^{r} \mathbf{w}_{i}\left(z_{k}\right) \Upsilon_{i}$ and its inverse $\Upsilon_{\mathbf{w}\left(z_{k}\right)}^{-1}=\left(\sum_{i=1}^{r} \mathbf{w}_{i}\left(z_{k}\right) \Upsilon_{i}\right)^{-1}$, with delayed scheduling functions $\Upsilon_{\mathbf{w}\left(z_{k+1}\right)}=\sum_{m=1}^{r} \mathbf{w}_{m}\left(z_{k+1}\right) \Upsilon_{m}$, or depending on nonavailable variables $\Upsilon_{\omega\left(\zeta_{k}\right)}=\sum_{j=1}^{\rho} \omega_{j}\left(\zeta_{k}\right) \Upsilon_{j}$, and so on. Additionally, $A>0(<0)$ means that $A \in \mathbb{R}^{n \times n}$ is positive (negative) definite. An asterisk $(*)$ will be used in matrix expressions to denote the transpose of the symmetric element; for in-line expressions, it will denote the transpose of the terms on its left side:

$$
\begin{aligned}
{\left[\begin{array}{ll}
A & B^{T} \\
B & C
\end{array}\right] } & =\left[\begin{array}{cc}
A & (*) \\
B & C
\end{array}\right] \text { and } A+B+A^{T}+B^{T}+C \\
& =A+B+(*)+C .
\end{aligned}
$$

Hence, system (12) is shortly written as

$$
\mathscr{E}_{\mathbf{w}\left(z_{k}\right)} e_{k+1}=\left(\overline{\mathscr{A}}_{\mathbf{w}\left(z_{k}\right) \omega\left(\zeta_{k}\right)}-L\left(\widehat{x}_{k}, y_{k}\right) \overline{\mathscr{C}}_{\mathbf{w}\left(z_{k}\right) \omega\left(\zeta_{k}\right)}\right) e_{k} \text {. }
$$

The following lemmas are useful in order to derive LMI conditions for the design of $L\left(\hat{x}_{k}, y_{k}\right)$. The first one concerns a sum-relaxation scheme based on [59]; the second one allows avoiding the computation of $\mathscr{E}^{-1}\left(y_{k}\right)$ while adding slack variables [27].

Lemma 1 (see [59]). Let $\Upsilon_{i l}^{j m}=\left(\Upsilon_{i l}^{j m}\right)^{T}, \quad(i, l, m) \in\{1,2$, $\ldots, r\}^{3}, j \in\{1,2, \ldots, \rho\}$, be matrices of adequate dimensions. Then, $\Upsilon_{\mathbf{w}\left(z_{k}\right) \mathbf{w}\left(z_{k}\right) \mathbf{w}\left(z_{k+1}\right) \omega\left(\zeta_{k}\right)}<0$ holds if

$$
\frac{2}{r-1} \Upsilon_{i i}^{j m}+\Upsilon_{i l}^{j m}+\Upsilon_{l i}^{j m}<0
$$

for all $(i, l, m) \in\{1,2, \ldots, r\}^{3}, j \in\{1,2, \ldots, \rho\}$.

Lemma 2 (see [60]). Let $\xi \in \mathbb{R}^{n}, Q ⿻ Q^{T} \in \mathbb{R}^{n \times n}$, and $\mathscr{B} \in \mathbb{R}^{m \times n}, \operatorname{rank}(\mathscr{B})<n$; then, the following statements are equivalent:

(i) $\xi^{T} Q \xi<0, \forall \mathscr{B} \xi=0, \xi \neq 0$

(ii) $\exists \mathscr{Z} \in \mathbb{R}^{n \times m}: \mathbb{Q}+\mathscr{Z} \mathscr{B}+\mathscr{B}^{T} \mathscr{Z}^{T}<0$

Now, we are ready to establish LMI conditions for the stabilization of error system (9) at the origin $e=0$ via its exact convex representation (12).

\section{LMI-Based Stabilization of the Error System}

This section provides LMI conditions to compute $L\left(\widehat{x}_{k}, y_{k}\right)$. The developments are based on a convex Lyapunov function candidate (in the context of TS models, it has been introduced as nonquadratic Lyapunov function [61], in the context of fuzzy systems as fuzzy Lyapunov functions [62], in the context of LPV models, as parameter dependent Lyapunov functions [63]. Here, the name convex Lyapunov function is adopted due to its dependence on the convex functions $\left.\mathbf{w}_{i}\left(z_{k}\right)\right)$ :

$$
V(e)=e_{k}^{T} P_{\mathbf{w}\left(z_{k}\right)} e_{k}, \quad P_{\mathbf{w}\left(z_{k}\right)}=\sum_{i=1}^{r} \mathbf{w}_{i}\left(z_{k}\right) P_{i}
$$

with $P_{i} \in \mathbb{R}^{(n+p q+q) \times(n+p q+q)}: P_{i}>0, i \in\{1,2, \ldots, r\}$; its variation is

$$
\Delta V(e)=e_{k+1}^{T} P_{\mathrm{w}\left(z_{k+1}\right)} e_{k+1}-e_{k}^{T} P_{\mathrm{w}\left(z_{k}\right)} e_{k},
$$

which can be expressed as follows (without substituting the dynamics of estimation error (9)):

$$
\Delta V(e)=\left[\begin{array}{c}
e_{k} \\
e_{k+1}
\end{array}\right]^{T}\left[\begin{array}{cc}
-P_{\mathbf{w}}\left(z_{k}\right) & 0 \\
0 & P_{\mathbf{w}\left(z_{k+1}\right)}
\end{array}\right]\left[\begin{array}{c}
e_{k} \\
e_{k+1}
\end{array}\right] .
$$

Note that function (16) is convex and it only depends on available signals. With this in mind, the following result states LMI conditions for the design of the nonlinear gain $L\left(\widehat{x}_{k}, y_{k}\right)$.

Theorem 1. The origin $e=0$ of error system (9), with an exact convex representation (12), is asymptotically stable if there exist matrices $P_{l} \in \mathbb{R}^{(n+p q+q) \times(n+p q+q)}, N_{l} \in \mathbb{R}^{(n+p q+q) \times o}$, and $G_{l} \in \mathbb{R}^{(n+p q+q) \times(n+p q+q)}, l \in\{1,2, \ldots, r\}$ such that $P_{l}>0$ and the LMIs in (15) are satisfied with

$$
\Upsilon_{i l}^{j m}:=\left[\begin{array}{cc}
-P_{l} & (*) \\
G_{l} \overline{\mathscr{A}}_{i j}-N_{l} \overline{\mathscr{C}}_{i j} & -G_{l} \mathscr{E}_{i}-\mathscr{E}_{i}^{T} G_{l}^{T}+P_{m}
\end{array}\right],
$$

for all $(i, l, m) \in\{1,2, \ldots, r\}^{3}, j \in\{1,2, \ldots, \rho\}$. Then, the observer gain is computed as $L\left(\hat{x}_{k}, y_{k}\right)=G_{\mathbf{w}\left(z_{k}\right)}^{-1} N_{\mathbf{w}\left(z_{k}\right)}$. Moreover, any trajectory $e_{k}$ starting in the outermost Lyapunov level set $\left\{e: V\left(e_{k}\right) \leq c\right\} \subset \Omega_{x} \times \Omega_{\widehat{x}}, c>0$ goes to zero as time goes to infinity.

Proof. Recall the variation of the Lyapunov function expressed as in (18); thus, error system (9), with exact convex representation (12), is also expressed as

$$
\left[\overline{\mathscr{A}}_{\mathbf{w}\left(z_{k}\right) \omega\left(\zeta_{k}\right)}-L\left(\hat{x}_{k}, y_{k}\right) \overline{\mathscr{C}}_{\mathbf{w}\left(z_{k}\right) \omega\left(\zeta_{k}\right)}-\mathscr{E}_{\mathbf{w}\left(z_{k}\right)}\right]\left[\begin{array}{c}
e_{k} \\
e_{k+1}
\end{array}\right]=0 .
$$

By Lemma 2, (18) and (20) can be written together:

$$
\begin{aligned}
& {\left[\begin{array}{c}
Z_{1} \\
Z_{2}
\end{array}\right]\left[\overline{\mathscr{A}}_{\mathbf{w}\left(z_{k}\right) \boldsymbol{\omega}\left(\zeta_{k}\right)}-L\left(\hat{x}_{k}, y_{k}\right) \overline{\mathscr{C}}_{\mathbf{w}\left(z_{k}\right) \boldsymbol{\omega}\left(\zeta_{k}\right)}-\mathscr{E}_{\mathbf{w}\left(z_{k}\right)}\right]} \\
& \quad+(*)+\left[\begin{array}{cc}
-P_{\mathbf{w}\left(z_{k}\right)} & 0 \\
0 & P_{\mathbf{w}\left(z_{k+1}\right)}
\end{array}\right]<0 .
\end{aligned}
$$

Hence, by choosing $Z_{1}=0$ and $Z_{2}=G_{\mathbf{w}\left(z_{k}\right)}$ and the definition $\quad L\left(\widehat{x}_{k}, y_{k}\right)=G_{\mathbf{w}\left(z_{k}\right)}^{-1} N_{\mathbf{w}\left(z_{k}\right)}, G_{\mathbf{w}\left(z_{k}\right)} \in$ $\mathbb{R}^{(p q+q+n) \times(p q+q+n)}, N_{\mathbf{w}\left(z_{k}\right)} \in \mathbb{R}^{(p q+q+n) \times o}$, and we have 


$$
\Upsilon_{\mathbf{w}\left(z_{k}\right) \mathbf{w}\left(z_{k}\right) \mathbf{w}\left(z_{k+1}\right) \mathbf{w}\left(\zeta_{k}\right)}:=\left[\begin{array}{c}
-P_{\mathbf{w}\left(z_{k}\right)} \\
G_{\mathbf{w}\left(z_{k}\right)} \overline{\mathscr{A}}_{\mathbf{w}\left(z_{k}\right) \mathbf{\omega}\left(\zeta_{k}\right)}-N_{\mathbf{w}\left(z_{k}\right)} \overline{\mathscr{C}}_{\mathbf{w}\left(z_{k}\right) \mathbf{w}\left(\zeta_{k}\right)}-G_{\mathbf{w}\left(z_{k}\right)} \mathscr{E}_{\mathbf{w}\left(z_{k}\right)}+(*)+P_{\mathbf{w}\left(z_{k+1}\right)}
\end{array}\right]<0
$$

Finally, by means of relaxation Lemma 1, the desired result yields.

Remark 1. The speed convergence of observer (6) can be increased if the following condition is verified $\Delta V(e) \leq\left(\alpha^{2}-1\right) V(e), 0<\alpha \leq 1$ which can be translated into LMIs, that is, solving the LMIs in (15) with

$$
\Upsilon_{i l}^{j m}:=\left[\begin{array}{cc}
-\alpha^{2} P_{l} & (*) \\
G_{l} \overline{\mathscr{A}}_{i j}-\bar{N}_{l i j} & -G_{l} \mathscr{E}_{i}-\mathscr{E}_{i}^{T} G_{l}^{T}+P_{m}
\end{array}\right],
$$

for all $(i, l, m) \in\{1,2, \ldots, r\}^{3}, j \in\{1,2, \ldots, \rho\}$.

The following result provides LMI conditions for standard systems and its corresponding observer, i.e., (6) with $E\left(y_{k}\right)=I_{n}$, where $I_{n}$ is the identity matrix. Hence, the next result follows directly from Theorem 1 .

Corollary 1. The origin of error system (9), with $E\left(y_{k}\right)=I_{n}$, is asymptotically stable if there exist $P_{l} \in \mathbb{R}^{(n+p q+q) \times(n+p q+q) \text {, }}$ $N_{l} \in \mathbb{R}^{(n+p q+q) \times o}$, and $G_{l} \in \mathbb{R}^{(n+p q+q) \times(n+p q+q)}, l \in\{1,2, \ldots, r\}$ such that $P_{l}>0$ and LMIs in (15) hold with

$$
\Upsilon_{i l}^{j m}=\left[\begin{array}{cc}
-P_{l} & (*) \\
G_{l} \bar{A}_{i j}-N_{l} \bar{C}_{i j} & -G_{l}-G_{l}^{T}+P_{m}
\end{array}\right],
$$

for all $(i, l, m) \in\{1,2, \ldots, r\}^{3}, j \in\{1,2, \ldots, \rho\}$. The nonlinear observer gain $L\left(\widehat{x}_{k}, y_{k}\right)=G_{\mathbf{w}\left(z_{k}\right)}^{-1} N_{\mathbf{w}\left(z_{k}\right)}$. Moreover, any trajectory $e_{k}$ starting in the outermost Lyapunov level set $\{e: V(e) \leq c\} \subset \Omega_{x} \times \Omega_{\widehat{x}}, c>0$ goes to zero as time goes to infinity.

Proof. It follows directly by considering $E\left(y_{k}\right)=I_{n}$ from the developments in Theorem 1.
Remark 2. The methodology in previous approaches starts by computing a convex model of the given nonlinear one (standard or descriptor), and then, the estimation error is computed; if the problem involves unmeasurable scheduling variables, it is solved by considering the already defined scheduling functions. In contrast with those works, the methodology hereby presented begins by obtaining the nonlinear estimation error dynamics via factorizations; then, it employs the sector nonlinearity approach for writing an equivalent convex representation; thus, a fitter convex model is employed and the observer gain includes all the available signals.

Remark 3. In this work, a convex Lyapunov function such as the one introduced in [61] is employed; naturally delayed scheduling functions can be included similar to [25] or to the generalization [27]. For instance, following [25], the Lyapunov function candidate would be $V(e)=e_{k}^{T} P_{\mathrm{w}\left(z_{k-1}\right)} e_{k}$, $P_{\mathbf{w}\left(z_{k-1}\right)}=\sum_{m=1}^{r} \mathbf{w}_{m}\left(z_{k-1}\right) P_{m}$ together with the observer gain $L\left(\hat{x}_{k}, \hat{x}_{k-1}, y_{k}, y_{k-1}\right)=G_{\mathbf{w}\left(z_{k}\right) \mathbf{w}\left(z_{k-1}\right)} N_{\mathbf{w}\left(z_{k}\right) \mathbf{w}\left(z_{k-1}\right)}$.

Remark 4. The numerical complexity of Theorem 1 can be approximated by $\log _{10}\left(n_{d}^{3} n_{l}\right)$ where $n_{d}=r(n+p q+q)(0.5(n+p q+q+1)+n+p q+q+o)$ is the number of decision variables and $n_{l}=2 r^{3} \rho(n+p q+q)$ is the number of LMI rows [64]. Moreover, if the number LMI conditions in Theorem 1 is large, one can reduce its complexity by "judiciously eliminating" some convex functions either from the Lyapunov function or the observer gain.

In the case without unknown inputs $\left(d_{k}=0\right)$ in $(1)$, the states of the resulting system can be estimated by the following nonlinear observer:

$$
E\left(y_{k}\right) \widehat{x}_{k+1}=A\left(\hat{x}_{k}, y_{k}\right) \hat{x}_{k}+B\left(y_{k}\right) u_{k}+L\left(\hat{x}_{k}, y_{k}\right)\left(y_{k}-\hat{y}_{k}\right), \quad \hat{y}_{k}=C\left(\hat{x}_{k}, y_{k}\right) \hat{x}_{k},
$$

where $\widehat{x}_{k} \in \mathbb{R}^{n}$ is the observer state and $L\left(\hat{x}_{k}, y_{k}\right) \in \mathbb{R}^{n \times o}$ is the observer gain to be designed. Following the methodology previously presented, the error dynamics is

$$
E\left(y_{k}\right) e_{k+1}=\left(\bar{A}\left(x_{k}, \widehat{x}_{k}\right)-L\left(\hat{x}_{k}, y_{k}\right) \bar{C}\left(x_{k}, \widehat{x}_{k}\right)\right) e_{k},
$$

where $\bar{A}\left(x_{k}, \hat{x}_{k}\right) e_{k}=A\left(x_{k}\right) x_{k}-A\left(\hat{x}_{k}, y_{k}\right) \hat{x}_{k}$ and $\bar{C}\left(x_{k}\right.$, $\left.\hat{x}_{k}\right) e_{k}=C\left(x_{k}\right) x_{k}-C\left(\hat{x}_{k}, y_{k}\right) \hat{x}_{k}$. Thus, we have the next result.

Corollary 2. The origin of error system (26) is asymptotically stable if there exist $P_{l} \in \mathbb{R}^{n \times n}, N_{l} \in \mathbb{R}^{n \times o}$, and $G_{l} \in \mathbb{R}^{n \times n}$, $l \in\{1,2, \ldots, r\}$ such that $P_{l}>0$ and LMIs in (15) hold with

$$
\Upsilon_{i l}^{j m}=\left[\begin{array}{cc}
-P_{l} & (*) \\
G_{l} \bar{A}_{i j}-N_{l} \bar{C}_{i j} & -G_{l} E_{i}-E_{i}^{T} G_{l}^{T}+P_{m}
\end{array}\right]
$$

for all $(i, l, m) \in\{1,2, \ldots, r\}^{3}, j \in\{1,2, \ldots, \rho\}$. The nonlinear observer gain $L\left(\hat{x}_{k}, y_{k}\right)=G_{\mathbf{w}\left(z_{k}\right)}^{-1} N_{\mathbf{w}\left(z_{k}\right)}$. Moreover, any trajectory $e_{k}$ starting in the outermost Lyapunov level set $\{e: V(e) \leq c\} \subset \Omega_{x} \times \Omega_{\widehat{x}}, c>0$ goes to zero as time goes to infinity.

Proof. The proof follows a similar path than the one for Theorem 1, with a Lyapunov function candidate $V(e)=e_{k}^{T} P_{\mathbf{w}\left(z_{k}\right)} e_{k}, P_{i}>0, P_{i} \in \mathbb{R}^{n \times n}, i \in\{1,2, \ldots, r\}$, and the nonlinear error dynamics (26) expressed in a convex form as 


$$
\left[\bar{A}_{\mathbf{w}\left(z_{k}\right) \omega\left(\zeta_{k}\right)}-L\left(\hat{x}_{k}, y_{k}\right) \bar{C}_{\mathbf{w}\left(z_{k}\right) \omega\left(\zeta_{k}\right)}-E_{\mathbf{w}\left(z_{k}\right)}\right]\left[\begin{array}{c}
e_{k} \\
e_{k+1}
\end{array}\right]=0
$$

and by the use of Finsler's lemma, with $L\left(\hat{x}_{k}, y_{k}\right)=G_{\mathbf{w}\left(z_{k}\right)}^{-1} N_{\mathbf{w}\left(z_{k}\right)}, Z_{1}=0$, and $Z_{2}=G_{\mathbf{w}\left(z_{k}\right)}$, we have

$$
\Upsilon_{\mathbf{w}\left(z_{k}\right) \mathbf{w}\left(z_{k}\right) \mathbf{w}\left(z_{k+1}\right) \mathbf{w}\left(\zeta_{k}\right)}:=\left[\begin{array}{c}
-P_{\mathbf{w}\left(z_{k}\right)} \\
G_{\mathbf{w}\left(z_{k}\right)} \bar{A}_{\mathbf{w}\left(z_{k}\right) \boldsymbol{\omega}\left(\zeta_{k}\right)}-N_{\mathbf{w}\left(z_{k}\right)} \bar{C}_{\mathbf{w}\left(z_{k}\right) \boldsymbol{\omega}\left(\zeta_{k}\right)}-G_{\mathbf{w}\left(z_{k}\right)} E_{\mathbf{w}\left(z_{k}\right)}+(*)+P_{\mathbf{w}\left(z_{k+1}\right)}
\end{array}\right]<0 .
$$

Finally, by means of relaxation Lemma 1, we conclude the proof.

Recall that most of the previous works do not consider scheduling functions depending on unmeasurable variables $[25,27]$ nor descriptor systems of form (1) [25].

\section{Actuator Fault Detection and Estimation}

In this section, an application of the UI observer developed above is employed in order to estimate and detect actuator faults $[46,47]$. Thus, let us now consider nonlinear discrete-time descriptor system (1) with an actuator fault:

$$
E\left(y_{k}\right) x_{k+1}=A\left(x_{k}\right) x_{k}+B\left(y_{k}\right) u_{k}+f_{a}\left(u_{k}, y_{k}\right)+D\left(y_{k}\right) d_{k}, \quad y_{k}=C\left(x_{k}\right) x_{k}
$$

where $f_{a}\left(u_{k}, y_{k}\right) \in \mathbb{R}^{m}$ represents the actuator fault vector; if it can be included inside of the unknown input, then (30) can be expressed as (1); in this case, conditions in Theorem 1 can be used. As customary in fault detection schemes, a residual signal based on the observer estimation of the unknown input is generated $[65,66]$ :

$$
r_{k}=\left\|\widehat{d}_{k}\right\| \text {. }
$$

The residual can be filtered to get a clear signal. The estimation of the fault can be done once the fault is considered as an unknown input [67].

Remark 5. It is possible to apply a mix of $H_{\infty}$ and $H_{-}$performances, for instance, $H_{\infty}$ attenuation in order to make residual (31) robust to uncertainties, noise, and the fault and the index $H_{-}$index in order to make more sensitive the detection of the fault $[68,69]$.

\section{Examples}

In this section, two examples are presented. The first one is intended to compare the performance of our proposal in contrast with recent approaches while the second one illustrates the estimation of actuator faults in the train system. The LMI conditions have been implemented in YALMIP
[70] with SeDuMi [71], while simulations have been run in Simulink for MATLAB2019b.

Example 1. Consider descriptor system (1) with matrices

$$
\begin{aligned}
E\left(y_{k}\right) & =\left[\begin{array}{cc}
2 & -\left(1+x_{1}^{2}\right)^{-1} \\
\left(1+x_{1}^{2}\right)^{-1} & 1
\end{array}\right], \\
A\left(x_{k}\right) & =\left[\begin{array}{ll}
x_{2}^{2} & -0.5 \beta \\
0.7 & \sin x_{1}
\end{array}\right], \\
B & =\left[\begin{array}{l}
0.1 \\
0.1
\end{array}\right], \\
D & =\left[\begin{array}{l}
0.2 \\
0.2
\end{array}\right], \\
C & =\left[\begin{array}{l}
1 \\
0
\end{array}\right]^{T},
\end{aligned}
$$

with $\beta>0$ is a known parameter. It is assumed that the unknown input has dynamics such as $(z-1)^{2} d_{k} \approx 0$, i.e., $\mathbf{d}_{k+1}=S \mathbf{d}_{k}$ with $S=\left[\begin{array}{cc}0 & 1 \\ -1 & 2\end{array}\right]$. In order to estimate both the state $x_{k}$ and the unknown input $d_{k}$, an extended system of form (4) and its corresponding observer (6) are constructed. Thus, the error dynamics $e_{k}=\chi_{k}-\widehat{\chi}_{k}$ can be computed as

$$
\mathscr{E}\left(y_{k}\right) e_{k+1}=\left[\begin{array}{c}
x_{1} x_{2}^{2}-0.5 \beta x_{2}+0.2 d_{1} \\
0.7 x_{1}+x_{2} \sin x_{1}+0.2 d_{1} \\
d_{2} \\
-d_{1}+2 d_{2}
\end{array}\right]-\left[\begin{array}{c}
\widehat{x}_{1} \hat{x}_{2}^{2}-0.5 \beta \widehat{x}_{2}+0.2 \hat{d}_{1} \\
0.7 \widehat{x}_{1}+\widehat{x}_{2} \sin \widehat{x}_{1}+0.2 \widehat{d}_{1} \\
\widehat{d}_{2} \\
-\widehat{d}_{1}+2 \widehat{d}_{2}
\end{array}\right]-L(\cdot)(y-\hat{y})
$$


Applying the factorization methodology in [41] and considering that $y=x_{1}$, we have $x_{2}^{2}-\widehat{x}_{2}^{2}=\left(x_{2}+\hat{x}_{2}\right)\left(x_{2}-\hat{x}_{2}\right)=$ $\left(x_{2}+\widehat{x}_{2}\right) e_{2}$ leading to final error dynamics

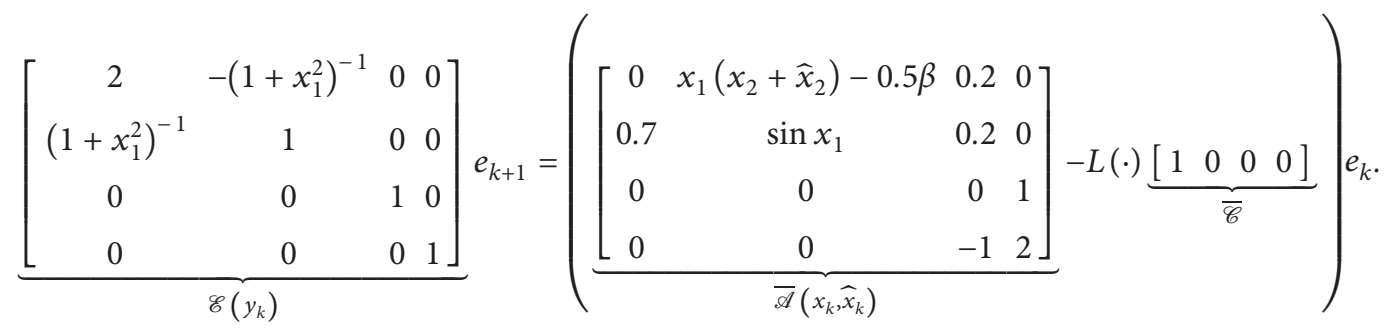

In order to synthesize the nonlinear observer gain via LMIs, it is necessary to express (34) in a convex form (12); to this end, let us consider the compact sets $\Omega_{x}=$ $\left\{\left|x_{1}\right| \leq 0.5,\left|x_{2}\right| \leq 0.8\right\}$ and $\Omega_{\widehat{x}}=\left\{\left|\widehat{x}_{2}\right| \leq 0.8\right\}$. Thus, the nonconstant terms and their bounds are $z_{1}=\left(1+x_{1}^{2}\right)^{-1}$ $\in[0.8,1], \quad z_{2}=\sin x_{1} \in[-0.4794,0.4794], \quad z_{3}=x_{1} \in$ $[-0.5,0.5], z_{4}=\hat{x}_{2} \in[-0.8,0.8]$, and $\zeta_{1}=x_{2} \in[-0.8,0.8]$ (nonavailable signals). The scalar convex functions are $\mathbf{w}_{i}(z)=w_{i_{1}}^{1}\left(z_{1}\right) w_{i_{2}}^{2}\left(z_{2}\right) w_{i_{3}}^{3}\left(z_{3}\right) w_{i_{4}}^{4}\left(z_{4}\right), \quad w_{0}^{i}\left(z_{i}\right)=\left(z_{i}^{1}-z_{i}\right) /$ $\left(z_{i}^{1}-z_{i}^{0}\right), \quad w_{1}^{i}\left(z_{i}\right)=1-w_{0}^{i}\left(z_{i}\right), \quad i \in\{1,2,3,4\}$, and $\omega_{j}(\zeta)=\omega_{j_{1}}^{1}\left(\zeta_{1}\right), \omega_{0}^{1}\left(\zeta_{1}\right)=\left(\zeta_{1}^{1}-\zeta_{1}\right) /\left(\zeta_{1}^{1}-\zeta_{1}^{0}\right), \omega_{1}^{1}=1-\omega_{0}^{1}$. The vertex matrices are

$$
\begin{aligned}
& \mathscr{E}_{i}=\left[\begin{array}{cccc}
2 & -z_{1}^{i_{1}} & 0 & 0 \\
z_{1}^{i_{1}} & 1 & 0 & 0 \\
0 & 0 & 1 & 0 \\
0 & 0 & 0 & 1
\end{array}\right] \\
& \overline{\mathscr{A}}_{i j}=\left[\begin{array}{cccc}
0 & z_{3}^{i_{3}}\left(\zeta_{1}^{j_{1}}+z_{4}^{i_{4}}\right)-0.5 \beta & 0.2 & 0 \\
0.7 & z_{2}^{i_{2}} & 0.2 & 0 \\
0 & 0 & 0 & 1 \\
0 & 0 & -1 & 2
\end{array}\right] \text {. }
\end{aligned}
$$

For example, if $i=6$ and $j=1$, we have $\mathbf{w}_{6}(z)=w_{0}^{1}\left(z_{1}\right) w_{1}^{2}\left(z_{2}\right) w_{0}^{3}\left(z_{3}\right) w_{1}^{4}\left(z_{4}\right), \omega_{1}(\zeta)=\omega_{0}^{1}\left(\zeta_{1}\right)$, and

$$
\begin{aligned}
\mathscr{E}_{6} & =\left[\begin{array}{cccc}
2 & -z_{1}^{0} & 0 & 0 \\
z_{1}^{0} & 1 & 0 & 0 \\
0 & 0 & 1 & 0 \\
0 & 0 & 0 & 1
\end{array}\right]=\left[\begin{array}{cccc}
2 & -0.8 & 0 & 0 \\
0.8 & 1 & 0 & 0 \\
0 & 0 & 1 & 0 \\
0 & 0 & 0 & 1
\end{array}\right], \\
\overline{\mathscr{A}}_{6,1} & =\left[\begin{array}{cccc}
0 & z_{3}^{0}\left(\zeta_{1}^{0}+z_{4}^{1}\right)-0.5 \beta & 0.2 & 0 \\
0.7 & z_{2}^{1} & 0.2 & 0 \\
0 & 0 & 0 & 1 \\
0 & 0 & -1 & 2
\end{array}\right]=\left[\begin{array}{cccc}
0 & -0.5 \beta & 0.2 & 0 \\
0.7 & 0.4794 & 0.2 & 0 \\
0 & 0 & 0 & 1 \\
0 & 0 & -1 & 2
\end{array}\right] .
\end{aligned}
$$

Several numerical tests have been performed in order to show the effectiveness of the proposal in contrast with recent works:

(i) LMIs in Theorem 1 have been run as well as the ones in [45], Theorem 2, with a Lyapunov function candidate (16) and decay rate $\alpha=0.95$ (see Remark 1 ), seeking feasibility for the largest $\beta>0$. It results that our proposal is feasible up to $\beta=0.863$, while the one in [45], Theorem 2 , is only up to $\beta=0.794$. Comparisons have been done with the same Lyapunov function (16) and under the same relaxation scheme. (ii) In terms of the number of decision variables, for this example, we have that numerical complexity for Theorem 1 is 12.86 while for [45] is 10.24 .

(iii) A UI observer uses the generalization in [27], and although it is for discrete-time descriptor systems, it cannot be applied as it only considers available scheduling variables.

(iv) If UI observer schemes for standard state-space models are to be applied $[33,39]$, then the inverse of the matrix $E\left(y_{k}\right)$ has to be computed. For instance, using conditions in Corollary 1 yields numerical problems because 65568 LMIs have to be solved. 
For illustration purposes, the LMI conditions in form (1) have been found feasible for $\beta=0.85$ and a decay rate $\alpha=0.95$, and some of the computed matrices are

$$
\begin{aligned}
& N_{1}=1 \times 10^{-4}\left[\begin{array}{c}
0.3502 \\
0.4455 \\
0.0786 \\
-0.0245
\end{array}\right] \text {, } \\
& G_{1}=1 \times 10^{-4}\left[\begin{array}{cccc}
0.4528 & 0.4482 & -0.1117 & -0.0147 \\
-0.4197 & 0.6839 & 0.0704 & -0.0772 \\
0.0656 & -0.3033 & 0.7189 & -0.5508 \\
-0.0796 & 0.1505 & -0.5751 & 0.4700
\end{array}\right] \text {, } \\
& N_{5}=1 \times 10^{-4}\left[\begin{array}{c}
0.4936 \\
0.5295 \\
0.0875 \\
-0.0429
\end{array}\right] \text {, } \\
& G_{5}=1 \times 10^{-4}\left[\begin{array}{cccc}
0.3710 & 0.5399 & -0.2236 & 0.0594 \\
-0.4458 & 0.6316 & 0.0105 & -0.0417 \\
0.0960 & -0.2168 & 0.6811 & -0.5264 \\
-0.0961 & 0.0893 & -0.5422 & 0.4492
\end{array}\right] \text {, } \\
& N_{16}=1 \times 10^{-4}\left[\begin{array}{c}
-0.1330 \\
0.7569 \\
0.1866 \\
-0.0911
\end{array}\right] \text {, } \\
& G_{16}=1 \times 10^{-4}\left[\begin{array}{cccc}
0.8122 & -0.3260 & 0.0464 & -0.0970 \\
-0.0814 & 0.9592 & -0.2018 & 0.0460 \\
-0.0773 & -0.1013 & 0.6744 & -0.5174 \\
-0.0047 & 0.0364 & -0.5419 & 0.4463
\end{array}\right] \text {. }
\end{aligned}
$$

A first simulation has been performed without unknown inputs, i.e., $d_{k}=0$ and the known input $u_{k}=0.4 \sin (0.6 k)$. Figure 1 shows evolution of the error signals converging asymptotically to the origin. A second simulation considers $u_{k}=0$, and the unknown input is defined as

$$
d_{k}= \begin{cases}0.4 k, & \text { if } 3 \leq k<5, \\ 0.8, & \text { if } 5 \leq k<12, \\ 0, & \text { otherwise, }\end{cases}
$$

and initial conditions $x(0)=[0.4-0.6]^{T}, \mathbf{d}(0)=\left[\begin{array}{ll}0 & 0\end{array}\right]^{T}$, $\widehat{x}(0)=\left[\begin{array}{ll}0 & 0\end{array}\right]^{T}$, and $\widehat{d}(0)=\left[\begin{array}{ll}0 & 0\end{array}\right]^{T}$. Figure 2 shows the state $x_{2}$ being adequately estimated while in Figure 3 , it can be seen that the observer effectively reconstructs the unknown input.

The next example provides an application of the UI observer hereby proposed for the task of actuator fault detection in a train system.

Example 2. This example provides a fault detection scheme via the proposed UI observer for a train system, and see Figure 4 for a diagram. Let us consider first the fault-free case, and thus, a continuous-time model is [72-74]

$$
\begin{aligned}
M \dot{v}(t) & =b_{T}(v(t)) u_{T}(t)-b_{B}(v(t)) u_{B}(t)-w(v(t))-g(\theta(p)), \\
\dot{p}(t) & =v(t),
\end{aligned}
$$

where $v(t)$ is the speed of the train, $p(t)$ is the position of the train, $b_{B}(v)$ is the maximum coefficient of the braking force, $u_{B}(t)$ is the relative braking force, $b_{T}(v)$ is the maximum coefficient of the traction force, $u_{T}(t)$ is the relative traction force, $g(\theta)$ is the force of declivity or external force, $w(v)$ is the friction force, and $M$ is the mass of the train. In practical cases, the declivity force is considered as $g(\theta(p))=$ $M g \sin (\theta(p))$, where $\theta(p)$ is the slope angle on the position $p(t)$ and $g$ is the gravity force [75]. In [76], the friction force $w(v)$ can be estimated via David's equation:

$$
w(v)=w_{0}+w_{1} v+w_{2} v^{2}
$$

where $w_{0}, w_{1}$, and $w_{2}$ are real coefficients that depend on the train characteristics and rail type; their values are unknown in the practice, but there exist some methods for their estimation [76]. By means of the Euler approximation $\dot{x}(t) \approx\left(x_{k+1}-x_{k}\right) / T_{s}$, where $T_{s}$ is the sampling time. Therefore, a discrete-time train system of (23) is

$$
\begin{aligned}
M v_{k+1}= & \left(b_{T}\left(v_{k}\right) u_{T_{k}}-b_{B}\left(v_{k}\right) u_{B_{k}}-w\left(v_{k}\right)\right. \\
& \left.-g\left(\theta\left(p_{k}\right)\right)\right) T_{s}+M v_{k}, \quad p_{k+1}=v_{k} T_{s}+p_{k} .
\end{aligned}
$$

The fault studied in this example is the jamming fault, and it may occur only during the braking. This fault depends on the adherence conditions between the wheel and the track, producing that when the brake locks the wheel, the wheel slides on the track $[68,74]$; in the real setup, the fault can occur depending on weather conditions. The main issue of a jamming fault is the impact directly on the measurements, producing a wrong estimate of both position and speed of the train. In Figure 5, it 


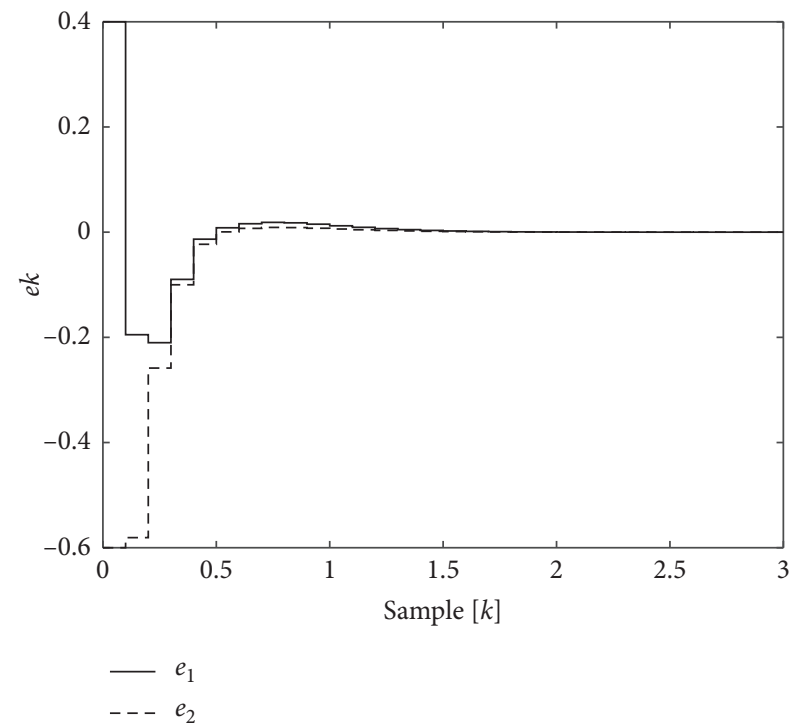

Figure 1: Error signal $e_{k}$ in the absence of unknown inputs.

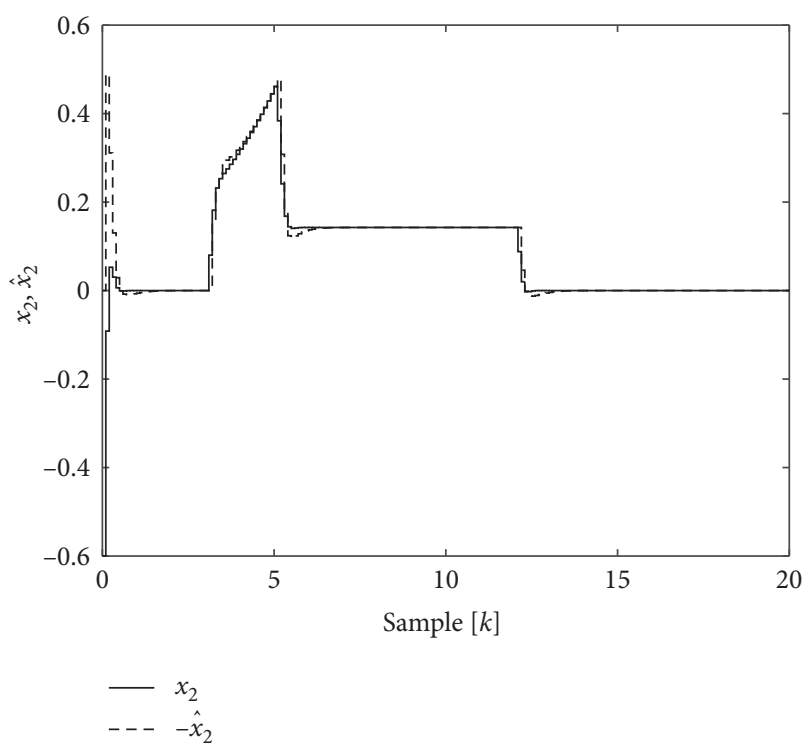

FIGURE 2: State $x_{2}$ and its estimation $\widehat{x}_{2}$.

can be seen that the speed $v_{k}$ under the fault is lower, and this is because the wheel is locked during the fault and the sensor is not able to provide the right measure and thus both the speed and position are erroneous; for example, the measured position differs 4.10 meters from the real position, see Figure 6. Therefore, this fault can be considered as an actuator fault or exogenous input, when it occurs, the control is inhibited [72-74]. The actuator fault to be considered is

$$
f_{a}\left(u_{T_{k}}, u_{B_{k}}\right)=-\left(b_{T}\left(v_{k}\right) u_{T_{k}}-b_{B}\left(v_{k}\right) u_{B_{k}}+\xi_{k}\right) f_{k},
$$

where $f_{k} \in[0,1]$ represents the grade of the fault; if $f_{k}=0$, then the system is fault-free; if $f_{k}=1$, a total fault occurs and

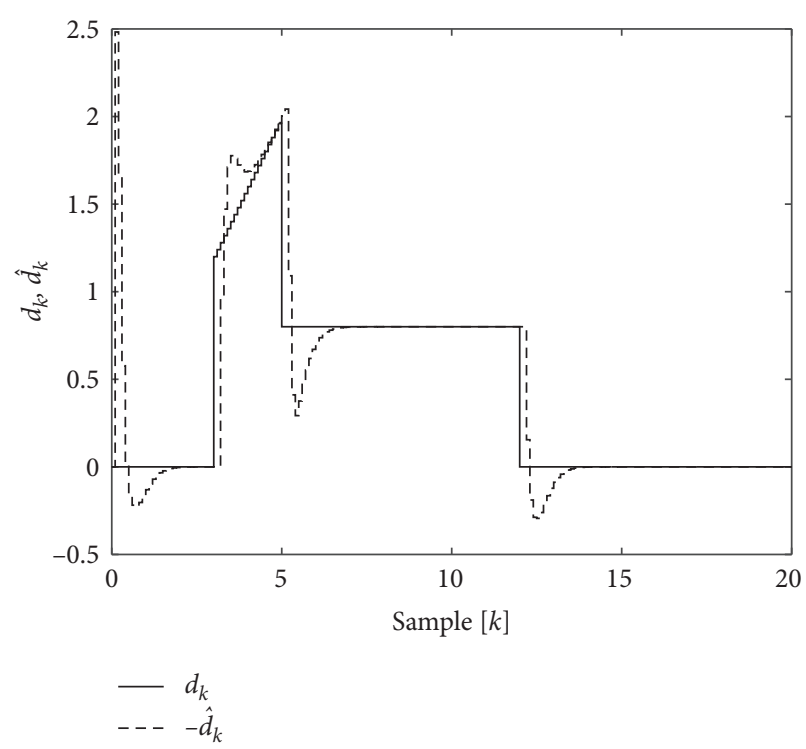

FIgURE 3: Unknown input $d_{k}$ and its estimation $\widehat{d}_{k}$.

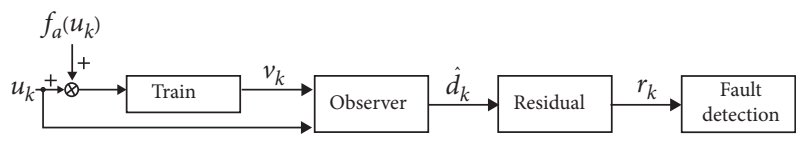

FIgURE 4: Fault detection scheme.

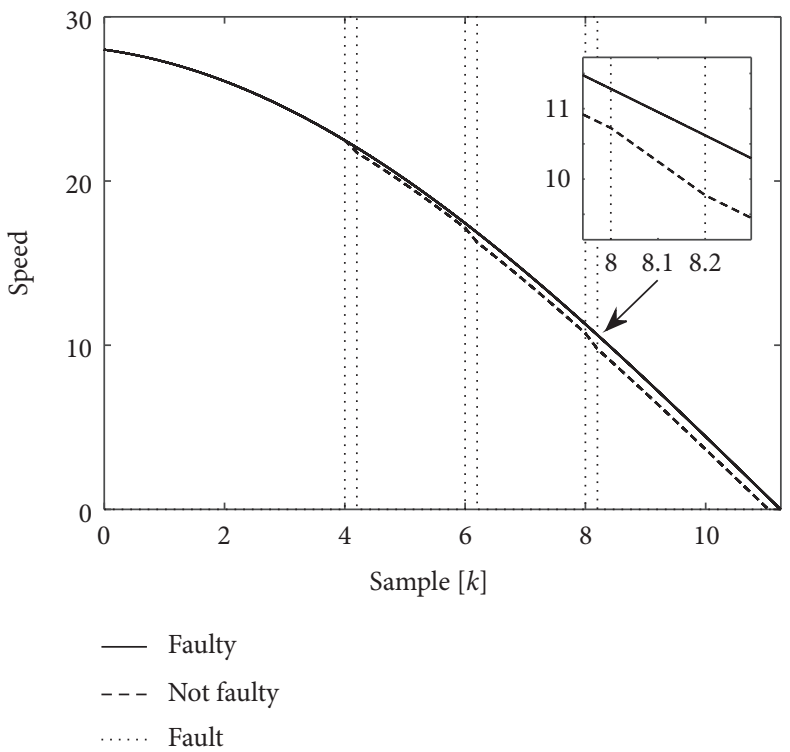

Figure 5: Comparison between the time evolution of the speed $v_{k}$ with fault and without fault.

the control is inhibited. The term $\xi_{k} \in \mathbb{R}$ represents a resistive force produced by the fault, and it is unknown [74].

As the previous examples, the unknown input is assumed to comply with $\mathbf{d}_{k+1}=S \mathbf{d}_{k}$; therefore, we have an augmented system of form (3) with $\chi=\left[\begin{array}{llll}v_{k} & p_{k} & d_{k} & d_{k+1}\end{array}\right]^{T}$ and matrices 


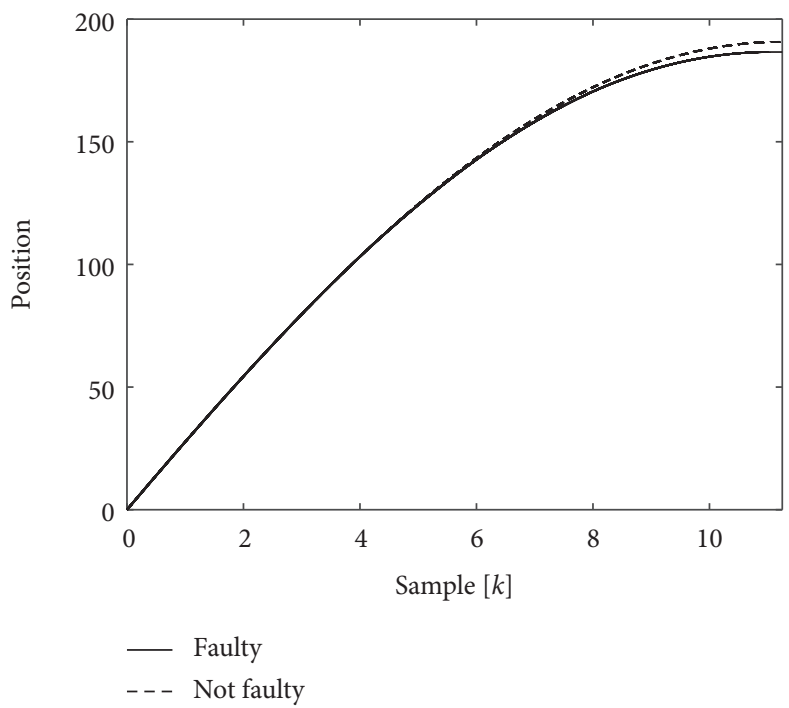

Figure 6: Comparison between the time evolution of the position $p_{k}$ with fault and without fault.

$$
\begin{aligned}
& \mathscr{E}=\left[\begin{array}{llll}
M & 0 & 0 & 0 \\
0 & 1 & 0 & 0 \\
0 & 0 & 1 & 0 \\
0 & 0 & 0 & 1
\end{array}\right], \\
& \mathscr{A}\left(y_{k}\right)=\left[\begin{array}{cccc}
\left(-w_{1}-w_{2} v_{k}\right) T_{s}+M & T_{s} g \frac{\left(\theta\left(p_{k}\right)\right)}{p_{k}} T_{s} & 0 \\
T_{s} & 1 & 0 & 0 \\
0 & 0 & 0 & 1 \\
0 & 0 & -1 & 2
\end{array}\right] \text {, } \\
& \mathscr{B}=\left[\begin{array}{l}
b \\
0 \\
0 \\
0
\end{array}\right], \\
& \mathscr{C}=\left[\begin{array}{l}
1 \\
0 \\
0 \\
0
\end{array}\right]^{T},
\end{aligned}
$$

and the parameter $b$ is a constant value for $b_{B}(v)$ and $b_{T}(v)$, producing a unique control signal $u_{k}$, where $u_{k}>0$ and $u_{k}<0$ correspond to $u_{T_{k}}$ and $u_{B_{k}}$, respectively. Let us consider the braking period, i.e., when the train is arriving to the station and there are no big slopes, we have $g\left(\theta\left(p_{k}\right)\right)=M g \sin \left(\theta\left(p_{k}\right)\right)$, with $\theta\left(p_{k}\right)=\beta p_{k} \in[-1,1]$, where $\beta$ is a constant.

Thus, with UI observer (4), the error dynamics $e_{k}=$ $\chi_{k}-\widehat{\chi}_{k}$ is

$$
\begin{aligned}
\mathscr{E} e_{k+1}= & {\left[\begin{array}{c}
\left(-w_{1} v_{k}-w_{2} v_{k}^{2}+d_{k}\right) T_{s}+M v_{k}-T_{s} M g \sin \left(\beta p_{k}\right) \\
T_{s} v_{k}+p_{k} \\
d_{k+1} \\
-d_{k}+2 d_{k+1}
\end{array}\right] } \\
& -\left[\begin{array}{c}
\left(-w_{1} \widehat{v}_{k}-w_{2} \widehat{v}_{k}^{2}+\widehat{d}_{k}\right) T_{s}+M \hat{v}_{k}-T_{s} M g \sin \left(\beta \widehat{p}_{k}\right) \\
T_{s} \widehat{v}_{k}+\widehat{p}_{k} \\
\widehat{d}_{k+1} \\
-\widehat{d}_{k}+2 \widehat{d}_{k+1}
\end{array}\right] \\
& -L(\cdot)(y-\hat{y}) .
\end{aligned}
$$

Applying the factorization in [41] and considering that $y_{k}=v_{k}$ (the only available signal is the velocity $v_{k}$ ), we have $v_{k}^{2}-\widehat{v}_{k}^{2}=\left(v_{k}+\widehat{v}_{k}\right) e_{1}=2 y_{k} e_{1}, e_{1}=v_{k}-\widehat{v}_{k}$, as for $\sin \left(\beta p_{k}\right)$ $-\sin \left(\beta \widehat{p}_{k}\right)$, two terms of the series Taylor are taken; then, the error dynamics yields:

$$
\mathscr{E} e_{k+1}=\left(\overline{\mathscr{A}}\left(y_{k}, \chi_{k}, \hat{\chi}_{k}\right)-L\left(y_{k}\right) \overline{\mathscr{C}}\right) e_{k},
$$

with matrices 


$$
\begin{aligned}
\overline{\mathscr{A}}\left(y_{k}, \chi_{k}, \widehat{\chi}_{k}\right) & =\left[\begin{array}{cccc}
-\left(w_{1}+2 w_{2} y_{k}\right) T_{s}+M & T_{s} M g\left(-1-\frac{\beta^{3}}{6}\left(p_{k}^{2}+p_{k} \widehat{p}_{k}+\widehat{p}_{k}^{2}\right)\right) & T_{s} & 0 \\
T_{s} & 1 & 0 & 0 \\
0 & 0 & 0 & 1 \\
0 & 0 & -1 & 2
\end{array}\right], \\
\overline{\mathscr{C}} & =\left[\begin{array}{l}
1 \\
0 \\
0 \\
0
\end{array}\right] .
\end{aligned}
$$

Therefore, considering that $v_{k} \in[0,30](\mathrm{m} / \mathrm{s})$ and $p_{k}, \widehat{p}_{k} \in[0,600](\mathrm{m})$, we have that the nonconstant terms are $z=v_{k} \in[0,30] \quad$ (available), $\zeta_{1}=p_{k}^{2} \in[0,360000]$, $\zeta_{2}=p_{k} \in[0,600], \quad \zeta_{3}=\widehat{p}_{k} \in[0,600], \quad$ and $\quad \zeta_{4}=\widehat{p}_{k}^{2} \epsilon$ $[0,360000]$ (nonavailable signals). The scheduling functions are defined as $\omega_{j}(\zeta)=\omega_{j_{1}}^{1}\left(\zeta_{1}\right) \omega_{j_{2}}^{2}\left(\zeta_{2}\right) \omega_{j_{3}}^{3}\left(\zeta_{3}\right) \omega_{j_{4}}^{4}\left(\zeta_{4}\right)$, $\omega_{0}^{j}\left(\zeta_{j}\right)=\left(\zeta_{j}^{1}-\zeta_{j}\right) /\left(\zeta_{j}^{1}-\zeta_{j}^{0}\right), \quad \omega_{1}^{j}=1-\omega_{0}^{j}\left(\zeta_{j}\right), \quad$ and $\mathbf{w}_{1}(z)=\left(z^{1}-z\right) /\left(z^{1}-z^{0}\right), \mathbf{w}_{2}(z)=1-\mathbf{w}_{1}(z)$. The vertex matrices are

$$
\bar{A}_{i, j}=\left[\begin{array}{cccc}
-\left(w_{1}+2 w_{2} z^{i}\right) T_{s}+M T_{s} M g\left(-1-\frac{\beta^{3}}{6}\left(\zeta_{1}^{j_{1}}+\zeta_{2}^{j_{2}} \zeta_{3}^{j_{3}}+\zeta_{4}^{j_{4}}\right)\right) & T_{s} & 0 \\
T_{s} & 1 & 0 & 0 \\
0 & 0 & 1 & 0 \\
0 & 0 & -1 & 2
\end{array}\right] \text {, }
$$

where $i=\{1,2\}$ and $j=\{1,2,3,4\}$. The LMI conditions in Theorem 1 have been found feasible with a decay rate $\alpha=0.7$ (see Remark 1), and the parameters are $M=408.5$ tons,
$w_{0}=3.525(\mathrm{~N}), \quad w_{1}=2.98 \times 10^{-} 3(\mathrm{~N} / \mathrm{m}), \quad w_{2}=4.575 \times$ $10^{-} 4\left(\mathrm{~N}^{2} / \mathrm{m}^{2}\right)$, and $T_{s}=0.001(\mathrm{~s})$. The computed matrices are 


$$
N_{1}=1 \times 10^{-5}\left[\begin{array}{c}
139.52295 \\
0.00157 \\
-0.00023 \\
0.00003
\end{array}\right]
$$$$
N_{2}=1 \times 10^{-5}\left[\begin{array}{c}
151.49720 \\
0.00172 \\
-0.00022 \\
0.00003
\end{array}\right] \text {, }
$$$$
G_{1}=1 \times 10^{-5}\left[\begin{array}{cccc}
0.34152 & 0.00284 & -0.00070 & 0.00035 \\
0.000007 & 0.0000001 & -0.00000003 & 0.00000002 \\
-0.000001 & -0.00000003 & 0.00000001 & -0.00000001 \\
0.0000008 & 0.00000002 & -0.00000001 & 0.000000008
\end{array}\right] \text {, }
$$$$
G_{2}=1 \times 10^{-5}\left[\begin{array}{cccc}
0.36992 & 0.00305 & -0.00071 & 0.00035 \\
0.000007 & 0.0000001 & -0.00000003 & 0.00000002 \\
-0.000001 & -0.00000003 & 0.00000001 & -0.00000001 \\
0.0000008 & 0.00000002 & -0.00000001 & 0.000000008
\end{array}\right] \text {, }
$$$$
P_{1}=1 \times 10^{-5}\left[\begin{array}{cccc}
125.55422 & 0.00267 & -0.00065 & 0.00033 \\
0.00267 & 0.00000009 & -0.00000003 & 0.00000002 \\
-0.00065 & -0.00000003 & 0.00000001 & -0.00000001 \\
0.00033 & 0.00000002 & -0.000000001 & 0.0000000075
\end{array}\right] \text {, }
$$$$
P_{2}=1 \times 10^{-5}\left[\begin{array}{cccc}
129.30379 & 0.00278 & -0.00068 & 0.00035 \\
0.00278 & 0.0000001 & -0.00000003 & 0.00000002 \\
-0.00068 & -0.00000003 & 0.00000001 & -0.00000001 \\
0.00035 & 0.00000002 & -0.00000001 & 0.000000007
\end{array}\right] \text {. }
$$

A simulation has been performed for initial conditions $v(0)=28, \quad p(0)=0, \quad \mathbf{d}(0)=\left[\begin{array}{ll}0 & 0\end{array}\right]^{T}, \quad \widehat{v}(0)=27.9$, $\widehat{p}(0)=0.05$, and $\widehat{\mathbf{d}}(0)=\left[\begin{array}{ll}0.05 & 0.01\end{array}\right]^{T}$, with a constant braking force $u_{k}=-0.5, \beta=0.0016$, and the unknown input $d_{k}=f_{a}\left(u_{k}\right)$, with $\xi_{k}=2 M$ and the fault occurrence

$$
f_{k}= \begin{cases}1, & \text { if } 4 \leq k \leq 4.2 \\ 1, & \text { if } 6 \leq k \leq 6.2 \\ 1, & \text { if } 8 \leq k \leq 8.2 \\ 0, & \text { otherwise. }\end{cases}
$$

As the previous example, first simulation has been performed without unknown inputs, i.e., $d_{k}=0$. Figure 7 shows the speed being adequately estimated while Figure 8 plots the Lyapunov function, whose signal is always positive and monotonously decreases to zero.

In the case where $d_{k}=f_{a}\left(u_{k}\right)$, in order to detect the fault, the unknown input estimation is used as residual $r_{k}=$ $\left|\widehat{d}_{k}\right|$ with a single threshold of $\epsilon=M / 2$. If the residual $r_{k}$ is bigger than $\epsilon$, then the fault is occurring; otherwise, the fault has finished. Thus, we obtain a delay average of $0.003 \mathrm{sec}-$ onds to detect when the fault begins and 0.006 to detect when the fault ends. As we can see from Figure 9, our proposal is enough to detect when the fault occurs. 


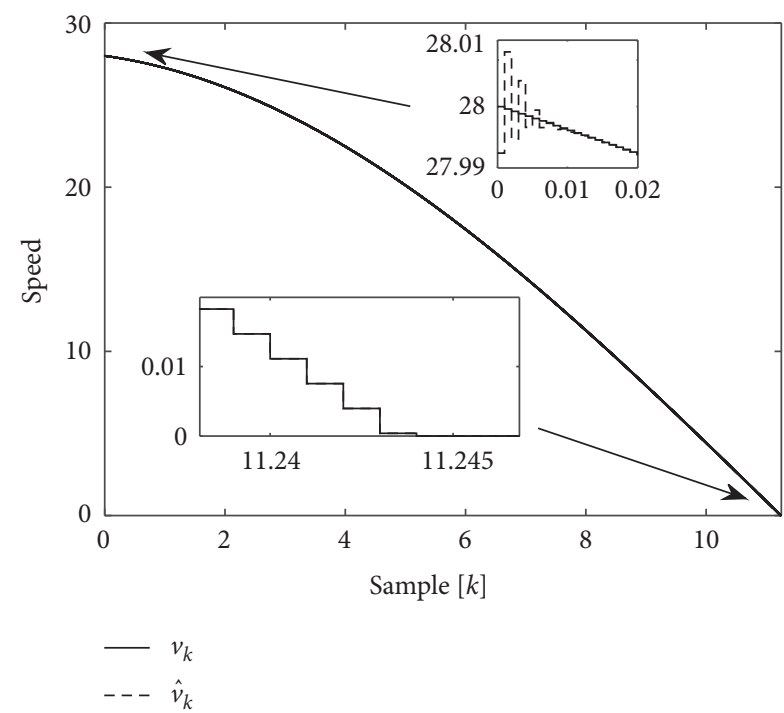

Figure 7: The time evolution of the speed $v_{k}$ and its estimation $\widehat{v}_{k}$.

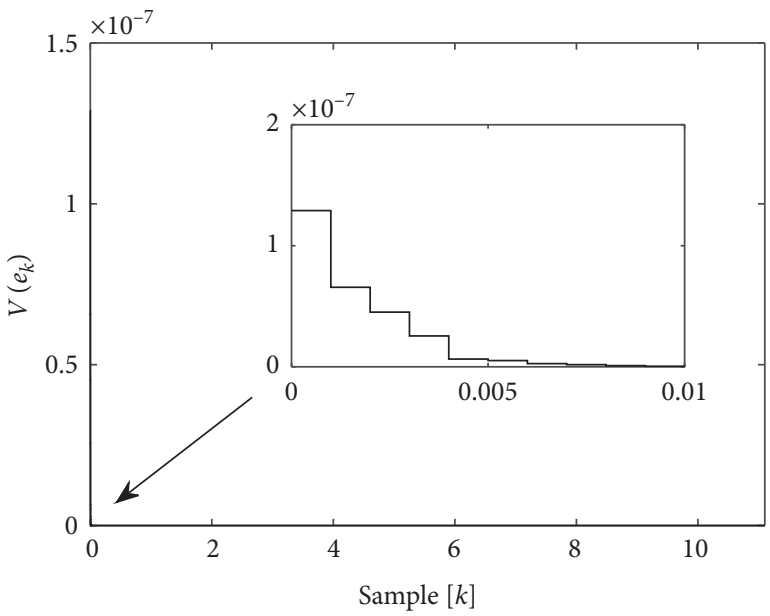

Figure 8: The time evolution of the Lyapunov function $V(e)$.

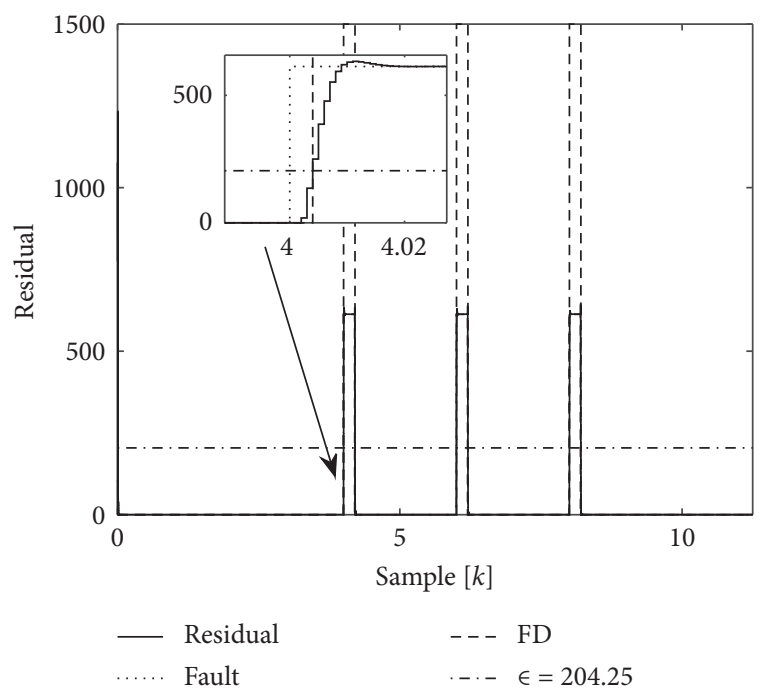

Figure 9: The time evolution of the residual $r_{k}$ and the fault detection.

\section{Conclusions}

It has been presented a methodology for the design of unknown input observers for discrete-time nonlinear descriptor systems. It is based on algebraic rearrangements and allows overcoming the problem of unmeasurable premise variables as to get a compatible error dynamic system with the direct Lyapunov method; thus, sufficient conditions in terms of LMIs have been obtained. The resulting UI observer is proven to be less conservative than those in the literature. Moreover, the proposed scheme has been employed to solve the detection and estimation of actuator faults. The advantages of the proposal have been illustrated via numerical example and applied to the fault detection in train systems.

\section{Data Availability}

No data were used to support this study. All the values to reproduce the results are included within the article.

\section{Conflicts of Interest}

The authors declare that there are no conflicts of interest regarding the publication of this paper.

\section{Acknowledgments}

This work was supported by CONACYT via the scholarship number 930683, the laboratory LANAVEX from the Universidad Politécnica de Pachuca, the Project ITSON-PROFAPI CA-18 2020-0038, and the postdoctoral fellowship PRODEP CA-18.

\section{References}

[1] D. Luenberger, "An introduction to observers," IEEE Transactions on Automatic Control, vol. 16, no. 6, pp. 596-602, 1971.

[2] Y. Guan and M. Saif, "A novel approach to the design of unknown input observers," IEEE Transactions on Automatic Control, vol. 36, no. 5, pp. 632-635, 1991.

[3] P. M. Frank, "Fault diagnosis in dynamic systems using analytical and knowledge-based redundancy," Automatica, vol. 26, no. 3, pp. 459-474, 1990.

[4] G.-R. Duan and R. J. Patton, "Robust fault detection using Luenberger-type unknown input observers-a parametric approach," International Journal of Systems Science, vol. 32, no. 4, pp. 533-540, 2001.

[5] R. Isermann, Fault-diagnosis Systems: An Introduction from Fault Detection to Fault Tolerance, Springer Science \& Business Media, Berlin, Germany, 2006.

[6] D. Ichalal, B. Marx, J. Ragot, and D. Maquin, "New fault tolerant control strategies for nonlinear Takagi-Sugeno systems," International Journal of Applied Mathematics and Computer Science, vol. 22, no. 1, pp. 197-210, 2012.

[7] Z. Dai, H. Zhou, J. Kang, and F. Wen, "The skewness of oil price returns and equity premium predictability," Energy Economics, vol. 94, Article ID 105069, 2021. 
[8] N. Kobayashi and T. Nakamizo, "An observer design for linear systems with unknown inputs," International Journal of Control, vol. 35, no. 4, pp. 605-619, 1982.

[9] M. Darouach, M. Zasadzinski, and S. J. Xu, "Full-order observers for linear systems with unknown inputs," IEEE Transactions on Automatic Control, vol. 39, no. 3, pp. 606-609, 1994.

[10] C.-C. Tsui, "A new design approach to unknown input observers," IEEE Transactions on Automatic Control, vol. 41, pp. 464-468, 1996.

[11] H. Khalil, Nonlinear Systems, Prentice Hall, Upper Saddle River, NJ, USA, 3rd edition, 2002.

[12] C. Edwards and C. P. Tan, "A comparison of sliding mode and unknown input observers for fault reconstruction," European Journal of Control, vol. 12, no. 3, pp. 245-260, 2006.

[13] D. Wang and K.-Y. Lum, "Adaptive unknown input observer approach for aircraft actuator fault detection and isolation," International Journal of Adaptive Control and Signal Processing, vol. 21, no. 1, pp. 31-48, 2007.

[14] W. Chen and M. Saif, "Unknown input observer design for a class of nonlinear systems: an LMI approach," in Proceedings of the 2006 American Control Conference, p. 5, IEEE, Minneapolis, MN, USA, June 2006.

[15] G. Besançon, "High-gain observation with disturbance attenuation and application to robust fault detection," Automatica, vol. 39, no. 6, pp. 1095-1102, 2003.

[16] K. Tanaka and H. Wang, Fuzzy Control Systems Design and Analysis: A Linear Matrix Inequality Approach, John Wiley \& Sons, New York, NY, USA, 2001.

[17] Z. Lendek, T. M. Guerra, R. Babuška, and B. De-Schutter, Stability Analysis and Nonlinear Observer Design Using Takagi-Sugeno Fuzzy Models, Springer-Verlag, Dordrecht, Netherlands, 2010.

[18] H. Ohtake, K. Tanaka, and H. O. Wang, "Fuzzy modeling via sector nonlinearity concept," in Proceedings of the Joint 9th IFSA World Congress and 20th NAFIPS International Conference, vol. 1, pp. 127-132, Vancouver, Canada, July 2001.

[19] T. Takagi and M. Sugeno, "Fuzzy identification of systems and its applications to modeling and control," IEEE Transactions on Systems, Man, and Cybernetics, vol. SMC-15, no. 1, pp. 116-132, 1985.

[20] J. S. Shamma, "An overview of lpv systems," in Control of Linear Parameter Varying Systems with Applications, pp. 3-26, Springer, Berlin, Germany, 2012.

[21] S. Boyd, L. E. Ghaoui, E. Feron, and V. Belakrishnan, "Linear matrix inequalities in system and control theory," SIAM: Studies In Applied Mathematics, vol. 15Philadelphia, PA, USA, 1994.

[22] C. Scherer, Linear Matrix Inequalities in Control Theory, Delf University, Delf, The Netherlands, 2004.

[23] K. Tanaka, T. Ikeda, and H. O. Wang, "Fuzzy regulators and fuzzy observers: relaxed stability conditions and LMI-based designs," IEEE Transactions on Fuzzy Systems, vol. 6, no. 2, pp. 250-265, 1998.

[24] P. Bergsten, R. Palm, and D. Driankov, "Observers for TakagiSugeno fuzzy systems," IEEE Transactions on Systems, Man and Cybernetics, Part B (Cybernetics), vol. 32, no. 1, pp. 114-121, 2002.

[25] T. M. Guerra, H. Kerkeni, J. Lauber, and L. Vermeiren, “An efficient Lyapunov function for discrete T-S models: observer design," IEEE Transactions on Fuzzy Systems, vol. 20, no. 1, pp. 187-192, 2012.

[26] T. Lei, Q. Song, and Y. Liu, "State estimation for discrete-time Takagi-Sugeno fuzzy systems with time-varying delays," Mathematical Problems in Engineering, vol. 2015, 2015.
[27] V. Estrada-Manzo, Z. Lendek, and T. M. Guerra, "Generalized LMI observer design for discrete-time nonlinear descriptor models," Neurocomputing, vol. 182, pp. 210-220, 2016.

[28] G.-P. Jiang, S.-P. Wang, and W.-Z. Song, "Design of observer with integrators for linear systems with unknown input disturbances," Electronics Letters, vol. 36, no. 13, pp. 1168-1169, 2000.

[29] D. Koenig and S. Mammar, "Design of proportional-integral observer for unknown input descriptor systems," IEEE Transactions on Automatic Control, vol. 47, no. 12, pp. 2057-2062, 2002.

[30] Z. Gao, X. Liu, and M. Z. Chen, "Unknown input observerbased robust fault estimation for systems corrupted by partially decoupled disturbances," IEEE Transactions on Industrial Electronics, vol. 63, pp. 2537-2547, 2015.

[31] D. Ichalal and S. Mammar, "On unknown input observers for LPV systems," IEEE Transactions on Industrial Electronics, vol. 62, no. 9, pp. 5870-5880, 2015.

[32] F. Xu, J. Tan, Y. Wang et al., "Generalized set-theoretic unknown input observer for LPV systems with application to state estimation and robust fault detection," International Journal of Robust and Nonlinear Control, vol. 27, pp. 38123832, 2017.

[33] B. Marx, D. Ichalal, J. Ragot, D. Maquin, and S. Mammar, "Unknown input observer for LPV systems," Automatica, vol. 100, pp. 67-74, 2019.

[34] H. R. Karimi and M. Chadli, "Robust observer design for Takagi-Sugeno fuzzy systems with mixed neutral and discrete delays and unknown inputs," Mathematical Problems in Engineering, vol. 2012, 2012.

[35] D. Ichalal, B. Marx, J. Ragot, and D. Maquin, "State estimation of Takagi-Sugeno systems with unmeasurable premise variables," IET Control Theory \& Applications, vol. 4, no. 5, pp. 897-908, 2010.

[36] A. Zemouche, M. Boutayeb, and G. I. Bara, "Observer design for nonlinear systems: an approach based on the differential mean value theorem," in Proceedings of the 44th IEEE Conference on Decision and Control and 2005 European Control Conference. CDC-ECC'05, pp. 6353-6358, IEEE, Seville, Spain, December 2005.

[37] T. M. Guerra, R. Márquez, A. Kruszewski, and M. Bernal, " $H_{\infty}$ LMI-based observer design for nonlinear systems via takagi-sugeno models with unmeasured premise variables," IEEE Transactions on Fuzzy Systems, vol. 26, no. 3, pp. 1498-1509, 2018.

[38] D. Ichalal, B. Marx, J. Ragot, and D. Maquin, "Design of observers for Takagi-Sugeno systems with immeasurable premise variables: an $L_{2}$ approach," in Proceedings of the 17th IFAC World Congress, pp. 2768-2773, Seoul, South Korea, July 2008.

[39] D. Ichalal, B. Marx, D. Maquin, and J. Ragot, "A method to avoid the unmeasurable premise variables in observer design for discrete time TS systems," in Proceedings of the 2016 IEEE International Conference on Fuzzy Systems (FUZZ-IEEE), pp. 2343-2348, Vancouver, Canada, July 2016.

[40] D. Ichalal, B. Marx, S. Mammar, D. Maquin, and J. Ragot, "How to cope with unmeasurable premise variables in TakagiSugeno observer design: dynamic extension approach," Engineering Applications of Artificial Intelligence, vol. 67, pp. $430-435,2018$.

[41] D. Quintana, V. Estrada-Manzo, and M. Bernal, "An exact handling of the gradient for overcoming persistent problems in nonlinear observer design via convex optimization techniques," Fuzzy Sets and Systems, 2020. 
[42] D. Luenberger, "Dynamic equations in descriptor form," IEEE Transactions on Automatic Control, vol. 22, no. 3, pp. 312-321, 1977.

[43] F. Lewis, D. Dawson, and C. Abdallah, Robot Manipulator Control: Theory and Practice, CRC Press, Boca Raton, FL, USA, 2003.

[44] M. Blandeau, V. Estrada-Manzo, T. M. Guerra, P. Pudlo, and F. Gabrielli, "Fuzzy unknown input observer for understanding sitting control of persons living with spinal cord injury," Engineering Applications of Artificial Intelligence, vol. 67, pp. 381-389, 2018.

[45] M. Blandeau, T.-M. Guerra, and P. Pudlo, "Application of a TS unknown input observer for studying sitting control for people living with spinal cord injury," in Control Applications for Biomedical Engineering Systems, Chap. 7, A. T. Azar, Ed., pp. 169-195, Elsevier, Amsterdam, Netherlands, 2020.

[46] J. Chen and H. Zhang, "Robust detection of faulty actuators via unknown input observers," International Journal of Systems Science, vol. 22, no. 10, pp. 1829-1839, 1991.

[47] J. Chen, R. J. Patton, and H.-Y. Zhang, "Design of unknown input observers and robust fault detection filters," International Journal of Control, vol. 63, no. 1, pp. 85-105, 1996.

[48] Z. Gao, C. Cecati, and S. X. Ding, "A survey of fault diagnosis and fault-tolerant techniques-Part I: fault Diagnosis with model-based and signal-based approaches," IEEE Transactions on Industrial Electronics, vol. 62, no. 6, pp. 3757-3767, 2015.

[49] W. Chen and M. Saif, "Fault detection and isolation based on novel unknown input observer design," in Proceedings of the 2006 American Control Conference, vol. 6-, Minneapolis, MN, USA, June 2006.

[50] P. Di Franco, G. Scarciotti, and A. Astolfi, "A globally stable algorithm for the integration of high-index differential-algebraic systems," IEEE Transactions on Automatic Control, vol. 65, no. 5, pp. 2107-2122, 2019.

[51] J. Arceo, M. Sánchez, V. Estrada-Manzo, and M. Bernal, "Convex stability analysis of nonlinear singular systems via linear matrix inequalities," IEEE Transactions on Automatic Control, vol. 64, pp. 1740-1745, 2018.

[52] L. Dai, Singular Control Systems, Vol. 118, Springer, Berlin, Germany, 1989.

[53] F. R. López-Estrada, D. Theilliol, C. M. Astorga-Zaragoza, J. C. Ponsart, G. Valencia-Palomo, and J. Camas-Anzueto, "Fault diagnosis observer for descriptor takagi-sugeno systems," Neurocomputing, vol. 331, pp. 10-17, 2019.

[54] S. Raghavan and J. K. Hedrick, "Observer design for a class of nonlinear systems," International Journal of Control, vol. 59, no. 2, pp. 515-528, 1994.

[55] A. Zemouche, M. Boutayeb, and G. I. Bara, "Observers for a class of Lipschitz systems with extension to performance analysis," Systems \& Control Letters, vol. 57, no. 1, pp. 18-27, 2008.

[56] M. Chadli and H. R. Karimi, "Robust observer design for unknown inputs Takagi-Sugeno models," IEEE Transactions on Fuzzy Systems, vol. 21, pp. 158-164, 2012.

[57] G. Phanomchoeng, R. Rajamani, and D. Piyabongkarn, "Nonlinear observer for bounded Jacobian systems, with applications to automotive slip angle estimation," IEEE Transactions on Automatic Control, vol. 56, no. 5, pp. 1163-1170, 2011.

[58] G. Wood and B. Zhang, "Estimation of the Lipschitz constant of a function," Journal of Global Optimization, vol. 8, pp. 91-103, 1996.

[59] H. D. Tuan, P. Apkarian, T. Narikiyo, and Y. Yamamoto, "Parameterized linear matrix inequality techniques in fuzzy control system design," IEEE Transactions on Fuzzy Systems, vol. 9, no. 2, pp. 324-332, 2001.

[60] M. Oliveira and R. Skelton, "Stability tests for constrained linear systems," in Perspectives in robust control, vol. 268 of Lecture Notes in Control and Information Sciences, pp. 241-257, Springer-Verlag, Berlin, Germany, 2001.

[61] T. M. Guerra and L. Vermeiren, "LMI-based relaxed nonquadratic stabilization conditions for nonlinear systems in the Takagi-Sugeno's form," Automatica, vol. 40, no. 5, pp. 823-829, 2004.

[62] K. Tanaka, T. Hori, and H. O. Wang, "A multiple Lyapunov function approach to stabilization of fuzzy control systems," IEEE Transactions on Fuzzy Systems, vol. 11, no. 4, pp. 582-589, 2003.

[63] J. Daafouz and J. Bernussou, "Parameter dependent Lyapunov functions for discrete time systems with time varying parametric uncertainties," Systems \& Control Letters, vol. 43, no. 5, pp. 355-359, 2001.

[64] X. Xie, D. Yue, T. Ma, and X. Zhu, "Further studies on control synthesis of discrete-time T-S fuzzy systems via augmented multi-indexed matrix approach," IEEE Transactions on Cybernetics, vol. 44, no. 12, pp. 2784-2791, 2014.

[65] P. M. Frank and X. Ding, "Survey of robust residual generation and evaluation methods in observer-based fault detection systems," Journal of Process Control, vol. 7, no. 6, pp. 403-424, 1997.

[66] V. Krishnaswami and G. Rizzoni, "A survey of observer based residual generation for FDI," IFAC Proceedings Volumes, vol. 27, no. 5, pp. 35-40, 1994.

[67] S. Li, H. Wang, A. Aitouche, Y. Tian, and N. Christov, "Active fault tolerance control of a wind turbine system using an unknown input observer with an actuator fault," International Journal of Applied Mathematics and Computer Science, vol. 28, no. 1, pp. 69-81, 2018.

[68] B. Aguiar, D. Berdjag, T.-M. Guerra, and B. Demaya, "Improving train position accuracy in case of wheel jamming faults," in Proceedings of the 2nd IFAC Workshop on Linear Parameter Varying Systems LPVS 2018, vol. 51, pp. 25-30, Florianópolis, Brazil, September 2018.

[69] J. L. Wang, G.-H. Yang, and J. Liu, “An LMI approach to $H_{-}$index and mixed/H_ $H_{-} \infty$ fault detection observer design," Automatica, vol. 43, pp. 1656-1665, 2007.

[70] J. Y. A. L. M. I. P. Lofberg, "A toolbox for modeling and optimization in MATLAB," in Proceedings of the 2004 IEEE International Symposium on Computer Aided Control Systems Design, pp. 284-289, Taipei, Taiwan, September 2004.

[71] J. F. Sturm, "Using SeDuMi 1.02, a MATLAB toolbox for optimization over symmetric cones," Optimization Methods and Software, vol. 11, no. 1-4, pp. 625-653, 1999. 
[72] Z. Mao, G. Tao, B. Jiang, and X.-G. Yan, "Adaptive compensation of traction system actuator failures for high-speed trains," IEEE Transactions on Intelligent Transportation Systems, vol. 18, no. 11, pp. 2950-2963, 2017.

[73] S. Liu, B. Jiang, Z. Mao, and S. X. Ding, "Adaptive backstepping based fault-tolerant control for high-speed trains with actuator faults," International Journal of Control, Automation and Systems, vol. 17, no. 6, pp. 1408-1420, 2019.

[74] B. Aguiar, D. Berdjag, B. Demaya, and T.-M. Guerra, "A robust and fault tolerant approach for automatic train stop control system design," in Proceedings of the 20th IFACPapersOnLine, vol. 50, no. 1, pp. 8549-8554, Toulouse, France, October 2017.

[75] P. G. Howlett, I. P. Milroy, and P. J. Pudney, "Energyefficient train control," Control Engineering Practice, vol. 2, no. 2, pp. 193-200, 1994.

[76] B. P. Rochard and F. Schmid, "A review of methods to measure and calculate train resistances," Proceedings of the Institution of Mechanical Engineers, Part F: Journal of Rail and Rapid Transit, vol. 214, no. 4, pp. 185-199, 2000. 